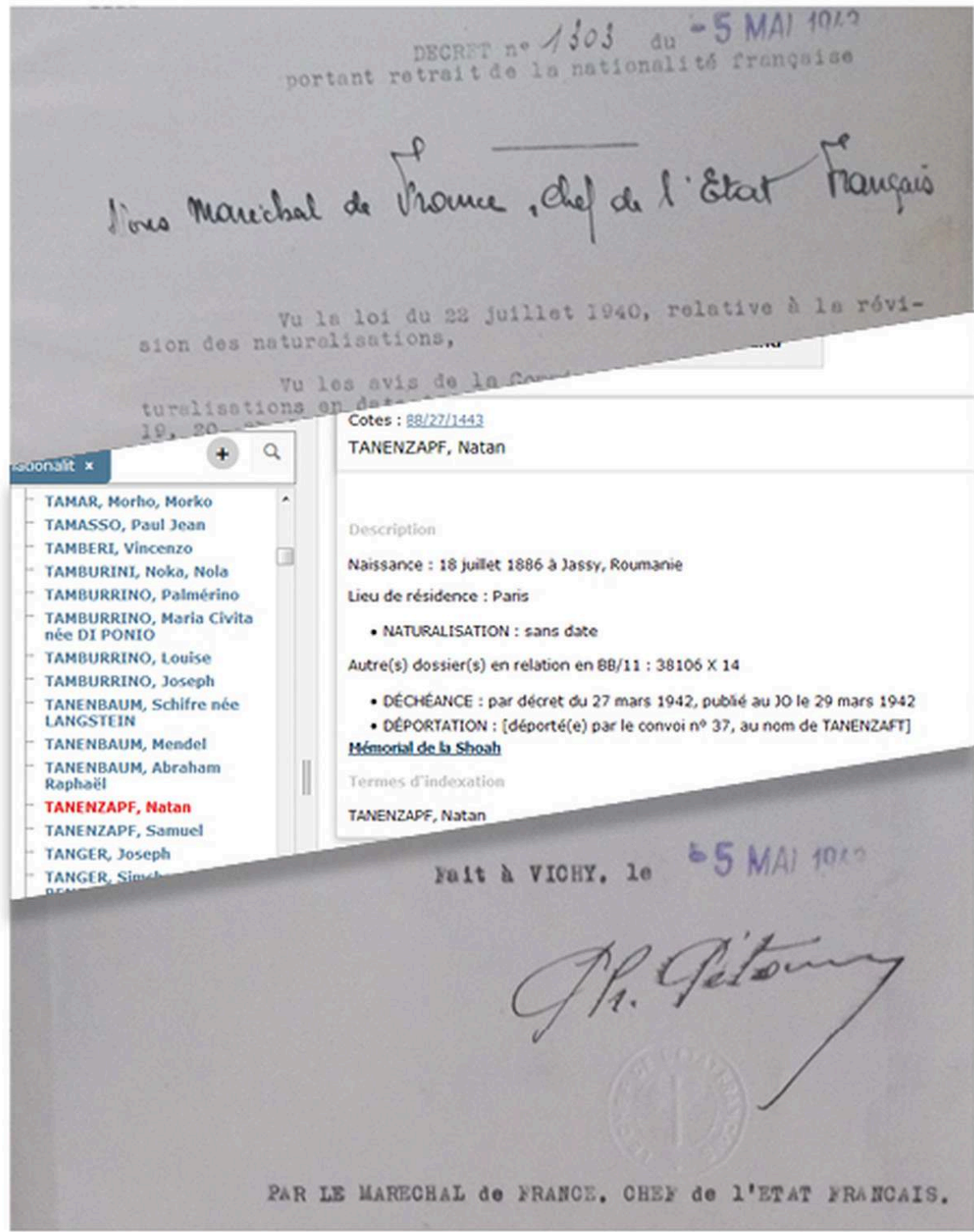

\title{
Connaître les dénaturalisés de Vichy
} La base Dénat, un nouvel outil et ses exploitations

\section{E. \\ TRER 5utur}




\section{Connaître les dénaturalisés de Vichy}

La base Dénat, un nouvel outil et ses exploitations

Thomas Lebée et Annie Poinsot (dir.)

DOI : 10.4000/books.pan. 1071

Éditeur : Publications des Archives nationales

Lieu d'édition : Pierrefitte-sur-Seine

Année d'édition : 2019

Date de mise en ligne : 2 octobre 2019

Collection : Actes

ISBN électronique : 9791036521683

\section{Q boooks}

http://books.openedition.org

\section{Référence électronique}

LEBÉE, Thomas (dir.) ; POINSOT, Annie (dir.). Connaitre les dénaturalisés de Vichy : La base Dénat, un nouvel outil et ses exploitations. Nouvelle édition [en ligne]. Pierrefitte-sur-Seine : Publications des Archives nationales, 2019 (généré le 10 septembre 2020). Disponible sur Internet : <http:// books.openedition.org/pan/1071>. ISBN : 9791036521683. DOI : https://doi.org/10.4000/books.pan. 1071.

Ce document a été généré automatiquement le 10 septembre 2020.

(C) Publications des Archives nationales, 2019

Conditions d'utilisation:

http://www.openedition.org/6540 
Cette journée d'étude a été organisé par les Archives nationales en 2018, suite à l'achèvement de l'enrichissement de la base Dénat, qui centralise les 15129 fiches d'individus naturalisés après 1927 et privés de la nationalité française par le régime de Vichy.

Cet outil exhaustif, lié à la base de données du Mémorial de la Shoah, documente la politique de privation des droits menée par l'État français envers des groupes qu'il excluait de la communauté nationale.

Il permet d'accéder aux dossiers de demande de naturalisation correspondants, très riches pour cerner « les dénaturalisés de Vichy » : situation familiale, enquêtes sur l'attitude politique et le patriotisme, parfois photographies et documents autographes.

Cette journée d'étude a dressé les premières exploitations qui peuvent être faites de ces sources sur les modalités d'organisation du retrait de la nationalité sous Vichy. Elle illustre la diversité des enquêtes qui peuvent être menées sur ces questions.

\section{THOMAS LEBÉE}

Chargé d'études documentaires

Responsable de fonds au pôle Justice (Archives nationales, direction des fonds, département de la Justice et de l'Intérieur)

\section{ANNIE POINSOT}

Chargée d'études documentaires principale

Responsable de fonds au pôle Justice (Archives nationales, direction des fonds, département de la Justice et de l'Intérieur) 


\section{SOMMAIRE}

\section{Préface}

Bernard Laguerre

Identifier, connaître et compter les victimes « absolues » de la dénaturalisation (1940-1944) Annie Poinsot et Bernard Raquin

Dénaturaliser : pourquoi, comment?

Que faire du fichier des dénaturalisés de Vichy?

2016 : un partenariat avec le Mémorial de la Shoah : échange des bases des dénaturalisés et des victimes de la Shoah

Dénaturaliser : qui, comment, pourquoi ?

Les Juifs sont-ils surreprésentés parmi les dénaturalisés?

Rôle du préfet, de ses services et des maires dans l'instruction des dossiers de dénaturalisation : l'exemple de la Meurthe-et-Moselle Jean-Claude Magrinelli

\section{Les dénaturalisations dans les colonies}

Laure Blévis et Claire Zalc

La « loi du 22 juillet 1940 » et les naturalisations dans l'Empire

Les dénaturalisations dans les colonies à partir de la base Dénat

Spécificités coloniales

Un nouveau mystère : la « loi » du 17 avril 1942

Les dossiers de dénaturalisation, nouvelle source pour l'histoire de la déportation en France Karen Taieb

La base des victimes de la Shoah en France

Les informations contenues dans les dossiers

Les recours

Conclusion et perspectives

Épilogue

Élisa Dupuis 


\section{NOTE DE L'ÉDITEUR}

Publication des actes de la journée d'étude organisée par les Archives nationales, en partenariat avec le Mémorial de la Shoah, à Pierrefitte-sur-Seine le 2 octobre 2018.

Consulter les inventaires issus de la base Dénat sur la salle des inventaires virtuelle des Archives nationales.

Les vidéos réalisées lors de cet événement sont visibles à partir de la page de chacune des communications. 


\section{Préface}

\section{Bernard Laguerre}

1 C'est en feuilletant au hasard les numéros du Journal officiel des années 1940 (ils étaient alors en accès libre à la bibliothèque de Sciences-Po) que je suis tombé pour la première fois sur un décret de dénaturalisation pris au titre de la loi du 22 juillet 1940: une longue liste de noms dont chacun indiquait un lieu et une date de naissance, une adresse, une profession, une date de naturalisation. Et ces décrets se retrouvaient, de mois en mois, de novembre 1940 à juin 1944 ; j'en ai alors compté 88.

2 De cette découverte et des recherches vers lesquelles elle m'a poussé, est né un article que j'ai publié, en 1988, sous le titre "Les dénaturalisés de Vichy ${ }^{1}$. Article plein de défauts, que j'écrirais certainement aujourd'hui autrement, mais qui avait pour lui de se pencher sur un épisode alors peu connu et passionnant de l'histoire de notre pays épisode qui, déjà alors, entrait en résonnance avec l'actualité - ce qui constituait à la fois un avantage et un inconvénient.

3 Trente ans après, que d'avancées! C'est ce que la lecture des actes de cette journée d'étude, consacrée à la base Dénat, permettra de découvrir. Le travail patient, et parfois ingrat, des historiens et des archivistes - on en lira des témoignages - a permis d'éclairer bien des points jusqu'ici demeurés dans l'ombre. Mais l'inconnu résiste et, faute d'accès aux archives de la Commission de révision des naturalisations, il est parti pour résister longtemps.

4 Ce qui résiste aussi, et résistera longtemps, c'est la stupéfaction qu'on ressent à la lecture de ces listes de noms et l'émotion qui nous étreint quand l'effort est fait de passer de la vision statistique à l'approche individuelle : tant de destins tordus, tant d'existences éprouvées, tant de vies brisées, notamment pour ces « victimes absolues » pour qui la dénaturalisation signifia la déportation.

5 Est-ce parce que j'ai quitté le monde des historiens? Je ne crois plus aux vertus de l'analogie historique. Celle-ci nous empêche plus souvent de penser le présent dans sa singularité qu'elle n'en éclaire le sens ou les contours. Mais l'histoire dit ce qui fut et établit la réalité de comportements et d'événements qui peuvent nous sembler insensés et incompréhensibles. C'est là son immense pouvoir. 
6 Ainsi des dénaturalisations de Vichy. Au rebours de ce qui est si souvent fait, il n'y a probablement rien à en tirer en termes d'analyse de l'actualité politique ou de combat partisan : aujourd'hui n'est pas hier. Mais savoir que ce fut fait et pouvoir de ses yeux voir ces longues listes de noms, ces dossiers consciencieusement annotés par des fonctionnaires scrupuleux, là est le coup de poing et l'ébranlement de nos certitudes. Là est la leçon terrible de l'histoire, sa vertu cathartique.

\section{NOTES}

1. « Les dénaturalisés de Vichy. 1940-1944 », Vingtième siècle. Revue d'histoire, 20, 1988, p. 3-15. 


\title{
Identifier, connaître et compter les victimes « absolues » de la dénaturalisation (1940-1944)
}

\author{
Annie Poinsot et Bernard Raquin
}

Ce média ne peut être affiché ici. Veuillez vous reporter à l'édition en ligne http://

Ce média ne peut être affiché ici. Veuillez vous reporter à l'édition en ligne http:// books.openedition.org/pan/1291

3 En 2008 était versé aux Archives nationales un fichier provenant de la sous-direction des naturalisations. Il s'agissait du fichier des « dénaturalisés de Vichy ». En l'absence d'archives liées au fonctionnement de la commission de révision des naturalisations mise en place entre 1940 et 1944, il fut décidé d'exploiter ce fichier de manière exhaustive au moyen d'une saisie systématique des informations contenues dans les fiches: la base Dénat était née. Après un long travail d'annotation des données, de vérifications, d'harmonisation, de conversion, de restitution de fiches manquantes, d'échanges avec le Mémorial de la Shoah, partenaire du projet, elle est aujourd'hui à la disposition des chercheurs sur le site des Archives nationales. Une mise en ligne est prévue en 2019. À terme, les plus de 8000 dossiers des 15000 " dénaturalisés de Vichy » seront numérisés avec la base Dénat. 


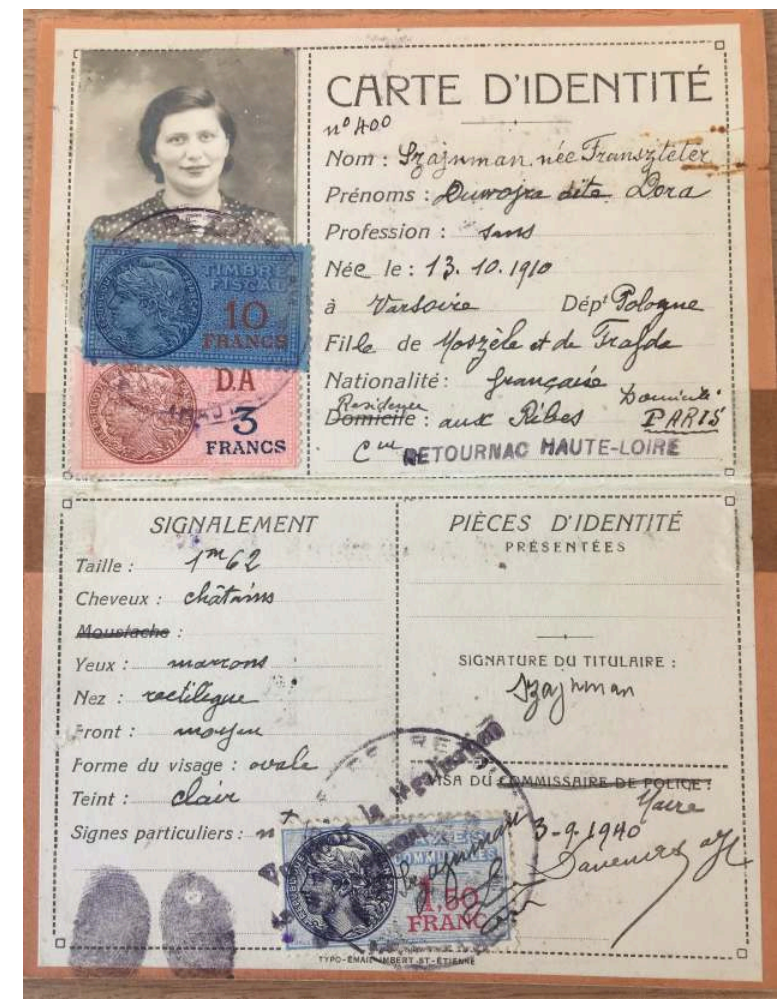

Pièce d'identité jointe à une demande de naturalisation (Archives nationales, 19770891/165, dossier $47388 \times 36)$

4 Après avoir rappelé quelques éléments du contexte entourant la loi du 22 juillet 1940 qui met en place la révision des naturalisations, nous reviendrons sur l'élaboration de la base Dénat avant de présenter les enseignements que l'on peut tirer de l'exploitation de ce nouvel instrument de recherche. Un éclairage particulier sera jeté sur la question des victimes « absolues » de cette procédure, à la fois dénaturalisées et déportées.

\section{Dénaturaliser : pourquoi, comment ?}

5 La loi du 22 juillet 1940 instaure la révision de « toutes les acquisitions de la nationalité française intervenues depuis la promulgation de la loi du 10 août 1927 sur la nationalité $»^{1}$. Objet de nombreuses critiques, surtout à droite et à l'extrême droite, la loi de 1927 est en effet accusée d'avoir fait des «Français de papier $»^{2}$ selon l'expression de l'Action française. La loi du 22 juillet 1940 est votée dix jours après l'arrivée à la Chancellerie de Raphaël Alibert, garde des Sceaux qui avait envisagé un retrait général de toutes les acquisitions de la nationalité française postérieures à 1927 avant de mettre en place ces dénaturalisations au cas par cas. ${ }^{3}$

6 C'est l'arrêté du 31 juillet 1940 qui fixe l'organisation et la composition de la commission de révision des naturalisations. Siégeant au ministère de la Justice, elle est rattachée au secrétariat général du gouvernement de Vichy. Présidée par Jean-Marie Roussel, conseiller d'État, la commission compte dix membres: des magistrats et des délégués de divers ministères. L'article 3 de la loi du 22 juillet 1940 stipule que les décrets de retraits de la nationalité sont pris sur le rapport du garde des Sceaux après avis de la commission de révision. Celle-ci siège à Paris, et non à Vichy, car il s'agit d'examiner des centaines de milliers de dossiers de naturalisation, qu'il est impossible 
de déplacer. Le bureau du Sceau au ministère de la Justice assure le secrétariat et communique les dossiers aux magistrats rapporteurs chargés de les étudier avant de présenter les cas lors des séances de la commission. Celle-ci émet un avis de maintien ou de retrait. Une enquête plus poussée, notamment auprès des préfets, peut être diligentée. Si l'avis de retrait est suivi par le garde des Sceaux, un décret de retrait de la nationalité ${ }^{4}$ est prononcé à l'encontre de ceux qui ont été « jugés indignes de conserver notre nationalité ». Ces retraits sont publiés en préfecture dans les tribunaux par voie d'affichage et insérés dans un journal local d'annonces légales. La gendarmerie ou l'autorité de police se présente également au domicile des intéressés pour notifier le retrait et réclamer la remise immédiate des ampliations de décrets de naturalisation et des cartes d'identité françaises de toute la famille ${ }^{5}$. La dénaturalisation peut être (et fut largement) « étendue à la femme et aux enfants " .

7 Le 21 septembre 1940 s'ouvre la première séance d'une commission de révision des naturalisations bien décidée à faire le tri entre les naturalisés qu'elle juge «acceptables» et ceux qu'elle juge «indésirables». Un travail colossal l'attend : examiner des dizaines de milliers de dossiers, décider du sort de centaines de milliers de personnes. Deux mois plus tard, pour accélérer l'examen des dossiers, sont créées trois sous-commissions et le 21 mars 1941, une nouvelle loi introduit le recours gracieux, qui permet, après réexamen devant la commission plénière, de revenir éventuellement sur un retrait ${ }^{7}$. La commission, lors de ses plus de 1400 séances entre novembre 1940 et mai 1944, aurait contribué à dénaturaliser 15154 hommes, femmes et enfants, selon les chiffres fournis par la sous-direction des naturalisations à la Libération ${ }^{8}$. Dans le même temps, 388 retraits auraient été rapportés (annulés), et 651 440 personnes maintenues dans la nationalité française.

8 L'ordonnance du 24 mai 1944 annule tous les décrets de retrait pris en application de la loi du 22 juillet 1940. Les dénaturalisés de Vichy sont juridiquement considérés par les autorités françaises comme n'ayant jamais cessé d'être Français.

\section{Que faire du fichier des dénaturalisés de Vichy?}

9 Le bureau du Sceau, au ministère de la Justice, assure donc le secrétariat de la commission de révision, c'est-à-dire le suivi des avis et des décisions. Pour ce faire, il met en place un fichier. Celui-ci, coté BB/27/1422 à 1445 aux Archives nationales, est une source essentielle pour étudier le fonctionnement de la commission de révision et mieux connaître les victimes de la procédure de retrait de la nationalité. Certes, les décrets de retraits sont publiés au Journal officiel et livrent des informations primordiales, mais l'accès ciblé aux dossiers de naturalisation, augmentés des pièces liées à la dénaturalisation, se fait ici grâce au numéro de dossier porté sur chaque fiche. Et ce sont bien ces dossiers conservés dans la série BB/11 des Archives nationales qui " témoignent » pour les hommes, femmes et enfants victimes de la dénaturalisation.

Plutôt qu'une numérisation, le choix a été fait de saisir intégralement le fichier dans une base de données permettant le croisement des informations. Cependant, à l'évidence, certaines fiches manquaient. Ainsi celles concernant les dénaturalisés compris dans le tout premier décret du $1^{\text {er }}$ novembre 1940, rassemblant plusieurs centaines de noms. Or, pour exploiter au mieux la base de données, il était indispensable de s'appuyer sur un travail exhaustif. La base a donc été complétée avec les noms manquants à partir du Journal officiel, sans omettre la mention que les 
quelques 450 fiches ainsi ajoutées étaient reconstituées. De même ont été saisies les fiches contenues dans l'article $\mathrm{BB} / 27 / 1421$ qui répertorie les personnes touchées par la procédure de déchéance de la nationalité française mise en place par la loi du 23 juillet 1940. Ces déchéances, d'un tout autre ordre que le retrait de la nationalité, frappent des Français non naturalisés ayant quitté le territoire national, autrement dit des résistants ou des politiques en exil, tels Charles de Gaulle ou Pierre Mendès France. Il a semblé utile de disposer d'un corpus des victimes des deux lois sur le retrait de la nationalité entre 1940 et 1944 : celle du 22 juillet 1940 (retraits visant les naturalisés d'après 1927) et celle du 23 juillet 1940 (déchéances visant les Français libres).

11 Le projet Dénat a toujours eu trois objectifs :

- effectuer un travail de description archivistique qui aboutisse à la production d'un outil orientant les chercheurs ;

- faire connaître un fichier qui recense les victimes d'une procédure peu ou mal connue ;

- valoriser comme objet «mémoriel » les dossiers de naturalisation et de dénaturalisation qui contiennent des documents tels que pièces d'état civil des pays d'origine, certificats de résidence et de scolarité, lettres manuscrites, photographies, toutes traces de vie de familles entières dont certaines disparurent en déportation.

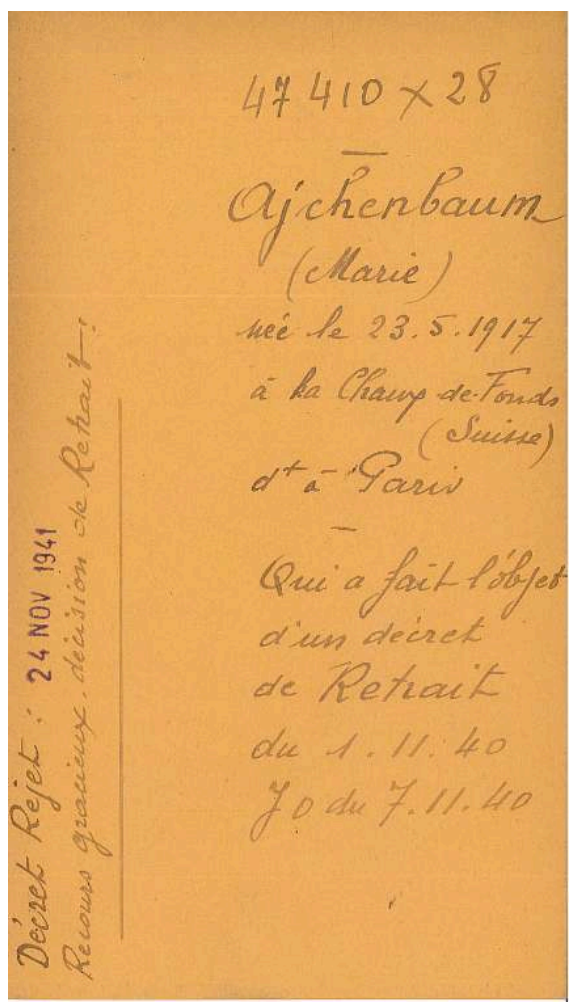

Fiche de retrait de la naturalisation de Marie Ajchenbaum (Archives nationales, BB/27/1422) et 


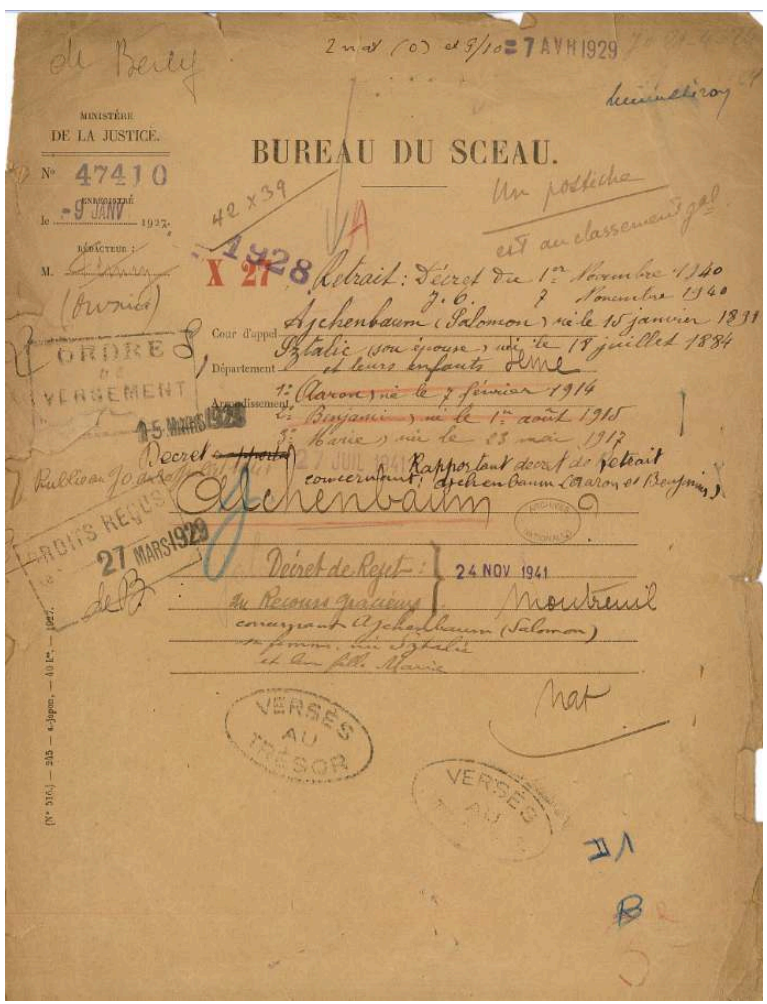

Couverture du dossier de naturalisation de la famille Ajchenbaum (Archives nationales, BB/11/10663, dossier $47410 \times 28$ )

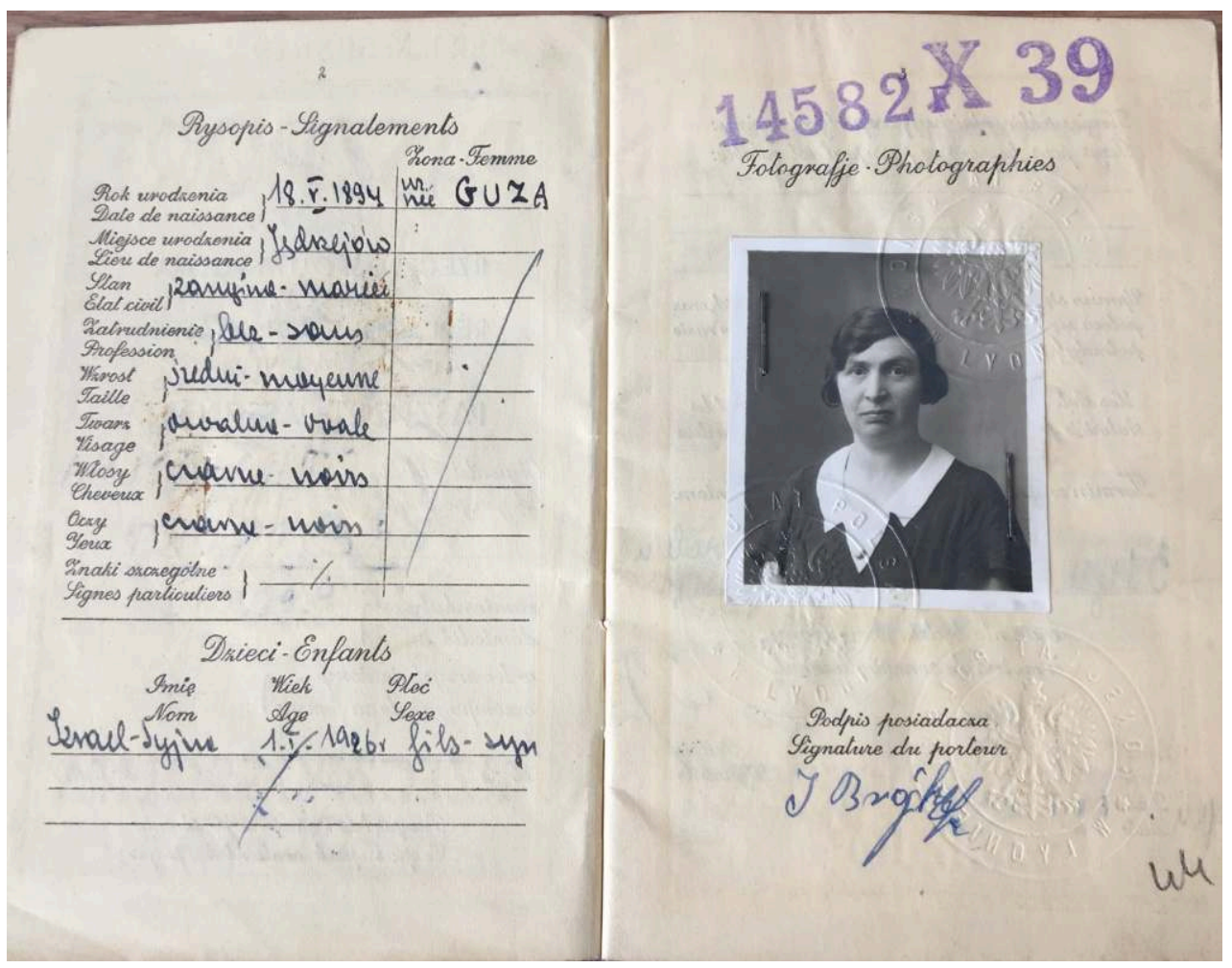

Passeport joint à un dossier de naturalisation (Archives nationales, 19770900/28, dossier 14582 X 39)

12 Ces trois objectifs ont présidé à l'élaboration de la base Dénat. 
La première étape a été le travail de dépouillement dans le système documentaire CinDoc, progiciel largement utilisé au ministère de la Culture et de la Communication. C'est ainsi que chaque renseignement indiqué sur une fiche fait l'objet d'un champ dans la base de données Dénat: nom, prénoms, date et lieu de naissance, date et type de naturalisation, date du décret de retrait et date de sa publication au Journal officiel, éventuel recours et date de décret de rejet de ce recours ou décret de retrait du retrait de la naturalisation, numéro et date de séance de la commission, numéro de dossier de naturalisation et des dossiers de naturalisation liés.

Il s'agissait ensuite de structurer cette base afin de rendre compte de la complexité du contenu des fiches. Dénat a donc été divisée en quatre ensembles :

- les éléments biographiques ;

\begin{tabular}{|l|}
\hline Perte de la nationalité \\
\hline COTE \\
\hline NOM \\
\hline Prénom(s) \\
\hline Nom de jeune fille \\
\hline Date de naissance \\
\hline Ville de naissance \\
\hline Pays de naissance \\
\hline Profession \\
\hline LIEU de RÉSIDENCE \\
\hline Sans domicile connu \\
\hline
\end{tabular}

\section{Retrait}

BB/27/1431

ABRAMOWICZ

Gitla

GOLDSTEIN

19/11/1897

Irena

Pologne

Meurthe-et-Moselle

- la procédure de naturalisation ;

\begin{tabular}{l} 
Date de naturalisation \\
\hline Type de naturalisation \\
\hline Renvoi au $\mathrm{N}^{\circ}$ du dossier en $\mathrm{BB} / 11$ \\
$\mathrm{~N}^{\circ}$ dossier(s) en relation en $\mathrm{BB} / 11$ \\
\hline
\end{tabular}

18/12/1929

Décret

$31541 \times 29$

$7242 \times 27$

- la procédure de retrait ;

\begin{tabular}{|l|}
\hline Date de séance \\
\hline$N^{\circ}$ de séance \\
\hline Commission plénière ou sous-commission(s) \\
\hline Date de retrait \\
\hline Date de publication au JO \\
\hline RECOURS gracieux \\
\hline ACCORD / Date du décret rapportant le rejet \\
\hline REFUS/Date du décret de rejet \\
\hline DÉCHÉANCE : Date \\
\hline Décision publiée au JO le : \\
\hline Loi Déchéance \\
\hline
\end{tabular}

$11 / 12 / 1940$

64

Non renseigné

21/03/1941

07/04/1941

OUI

04/12/1941

- la déportation, le cas échéant (informations reconstituées). 
15 La base ayant été alimentée par de multiples rédacteurs, s'en est suivie une deuxième étape consistant en un important travail d'harmonisation.

16 Comme on l'a vu, la base a été constituée à partir de trois ensembles distincts (les fiches de retrait conservées en $\mathrm{BB} / 27 / 1422$ à 1445 , les fiches de déchéances conservées en $\mathrm{BB} /$ 27/1421 et les fiches reconstituées à partir du Journal officiel). La totalité des entrées constituent un corpus cohérent sur la perte de nationalité, mais il devait demeurer possible de considérer et d'interroger chaque ensemble séparément.

\section{Déchéance}

Retrait

Retrait JO : Fiche manquante reconstituée à partir du Journal officiel

17 Or CinDoc génère des index permettant ensuite de faire des interrogations croisées sur chacun des ensembles et sur chacun des champs indexés.

18 L'étape suivante a été celle de la fusion de deux bases. Dans le cadre d'un partenariat, la base Dénat et celle des victimes de la Shoah en France, fournie par le Mémorial de la Shoah, ont fait l'objet d'un échange entre les deux institutions. Cet échange nous a permis d'identifier et de pointer les occurrences communes, un processus long et complexe.

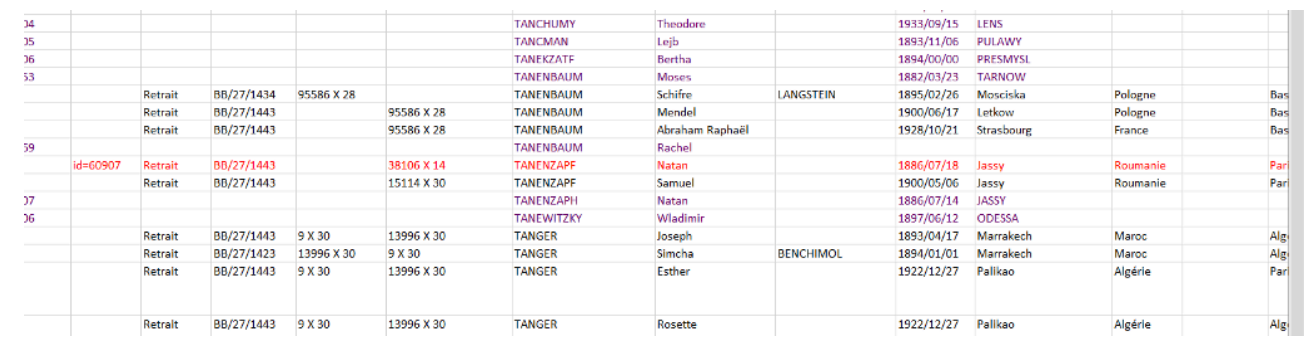

Tableau utilisé pour le repérage des doublons au moyens de tris successifs

\section{6 : un partenariat avec le Mémorial de la Shoah : échange des bases des dénaturalisés et des victimes de la Shoah}

19 Une fois le champ « ID-CDJC » (identifiant de la base des victimes de la Shoah en France) complété dans Dénat, il a été possible de créer le lien entre les deux bases. C'est ce lien qui permettra ensuite de passer de la base Dénat publiée dans la salle des inventaires virtuelle vers l'interface du Mémorial. 


\section{Retraits de nationalité}
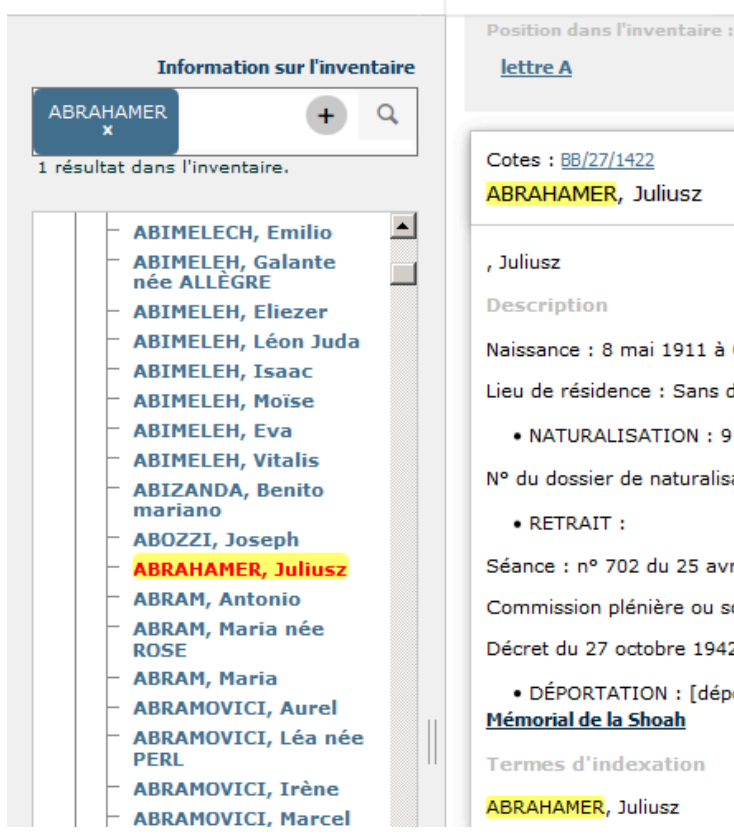

Interface de la notice de J. Abrahamer dans la maquette d'instrument de recherche créé à partir de Dénat et destiné à être publié dans la salle des inventaires virtuelle des Archives nationales.

Monsieur Julius ABRAHAMER né le 08/05/1911 à CRACOVIE.

DDocument(s) (1) Viqnette

Est inscrit(e) sur le Mur des Noms en tant que Monsieur Julius ABRAHAMER né(e) le 08/05/1911 à CRACOVIE (Pologne), déporté(e) par le convoi n 69 au départ de Drancy le 07/03/1944.

" Lettres du 17/03/1943, du dire...

Autre prénom connu "Julien".

A été résistant(e) sous le nom de $\underline{\text { Julien }}$ ABRAHAMER.

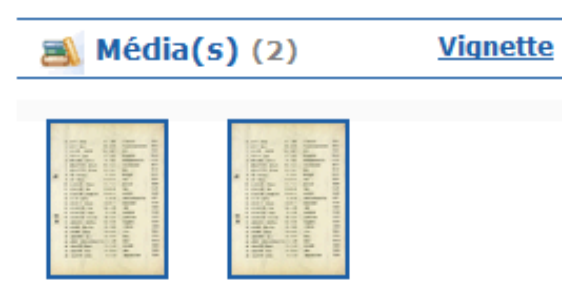

\section{Déportation Internement Fouilles Fiches de témoignages Autres victimes}

Monsieur Julius ABRAHAMER né le 08/05/1911 à CRACOVIE. Déporté à Auschwitz par le convoi $n^{\circ} 69$ au départ de Drancy le 07/03/1944. De profession Aide-comptable.

Monsieur Julius Abrahamer né en 1911 à Cracovie (Pologne). Déporté à Auschwitz par le convoi n 69 au départ de Drancy le 07/03/1944.

Décédé(e) en 1944

Notice du même J. Abrahamer sur le site du Mémorial de la Shoah (http://bdi.memorialdelashoah.org/ internet/jsp/core/MmsRedirector.jsp?id=261\&type=VICTIM)

Enfin, il a fallu convertir les données exportées de CinDoc en langage XML en conformité à la norme ISAD(G) et aux règles d'application de la DTD EAD (version 2002) en vigueur aux Archives nationales pour la publication de l'instrument de recherche dans la salle des inventaires virtuelle. 


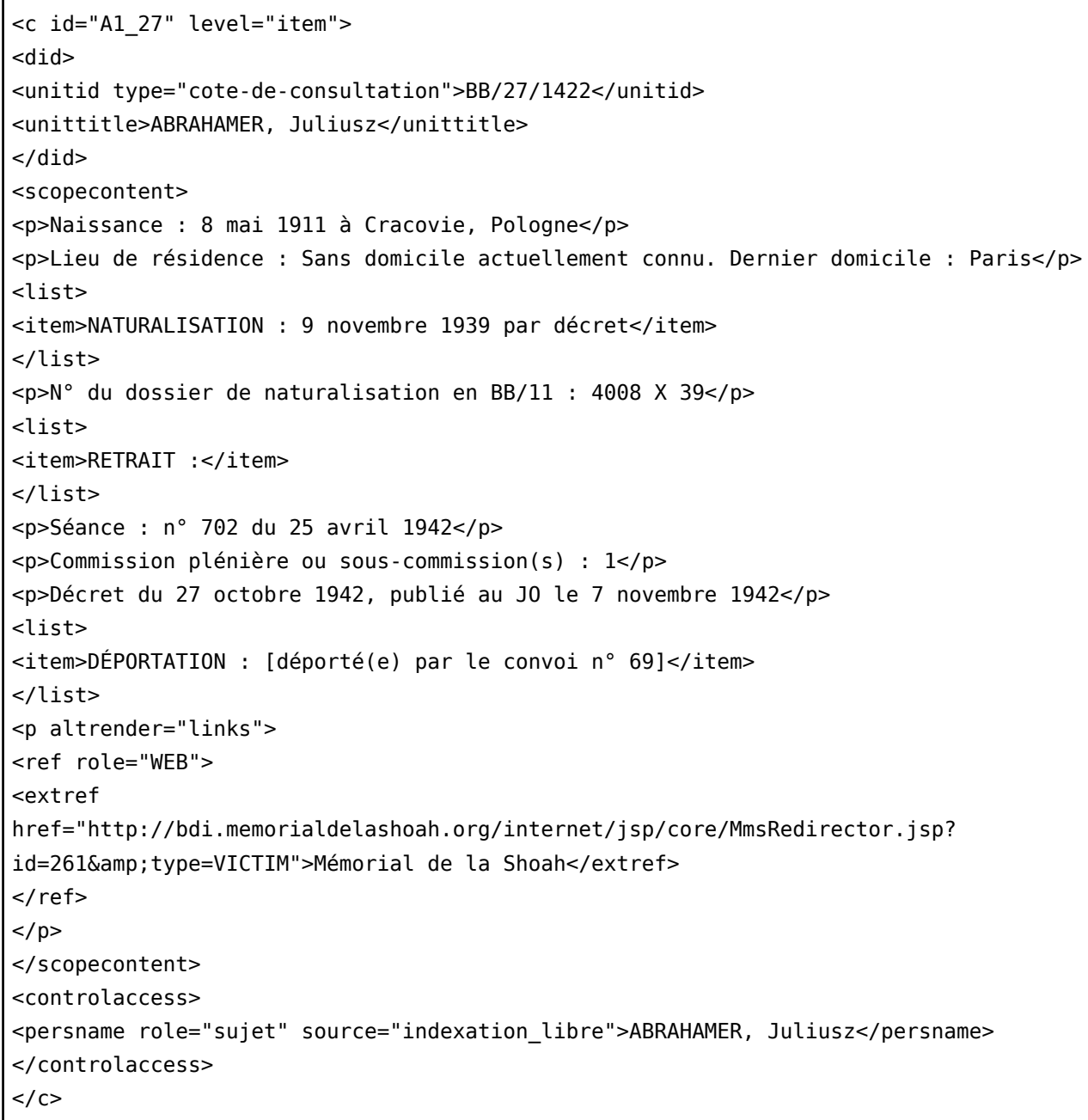

Une notice descriptive encodée

21 C'est à partir d'une base de données exhaustive et harmonisée que les premières exploitations statistiques ont pu être lancées.

\section{Dénaturaliser : qui, comment, pourquoi ?}

22 En considérant les différents graphiques et tableaux issus des données saisies, on mesure tous les enseignements que l'on peut tirer de la base Dénat: sur le fonctionnement de la commission de révision, mais aussi et surtout sur les dénaturalisés eux-mêmes. Dans le premier cas, à partir des dates et numéros de séance, des dates de décret, des numéros de sous-commissions, des recours acceptés ou rejetés, et dans le second cas avec des tris par pays et/ou par ville d'origine, par lieu de résidence, par âge même (voir ci-dessous). 


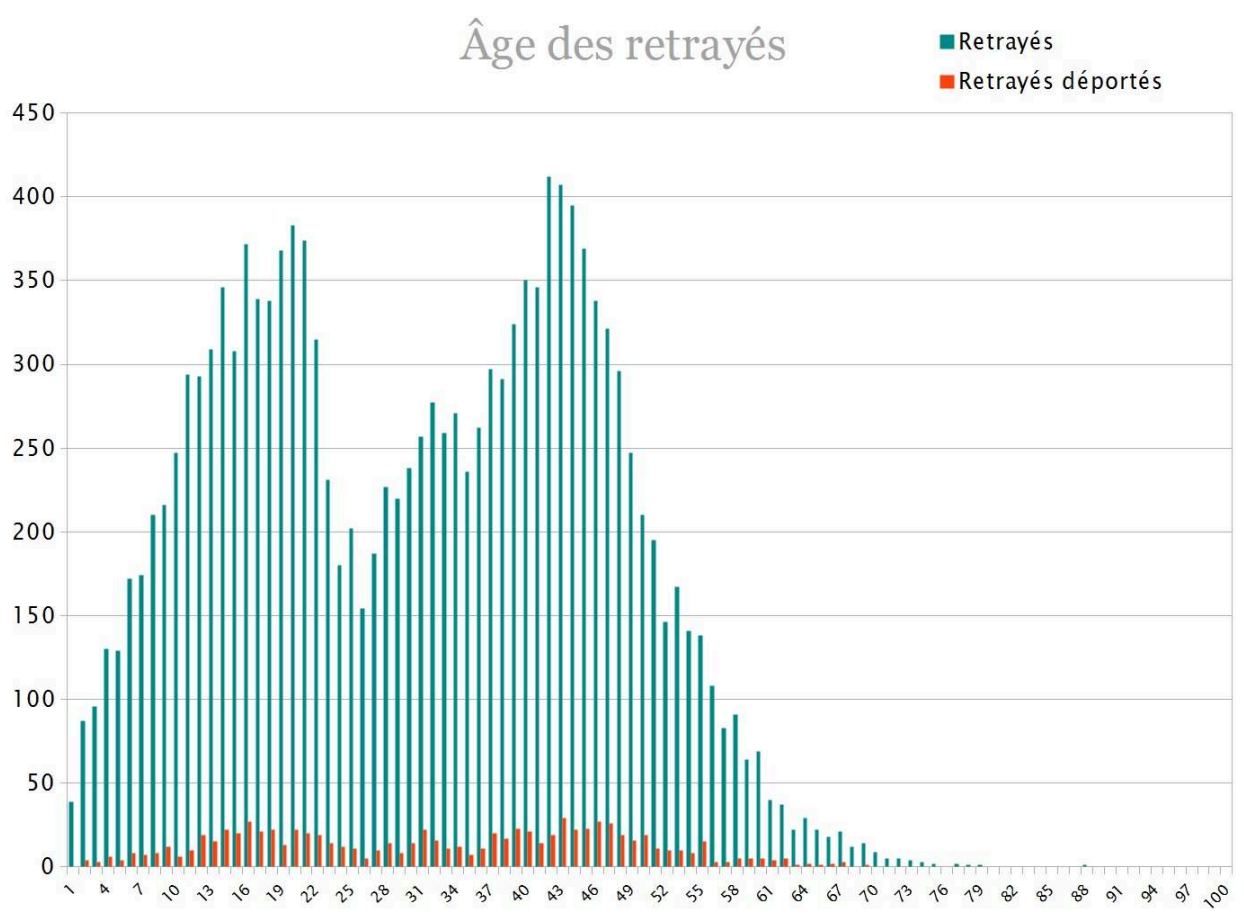

Nombre de retrayés et de retrayés déportés en fonction de leur âge

On constate ainsi que la France est le pays de naissance le plus représenté (les enfants nés en France ayant été dénaturalisés avec leurs parents).

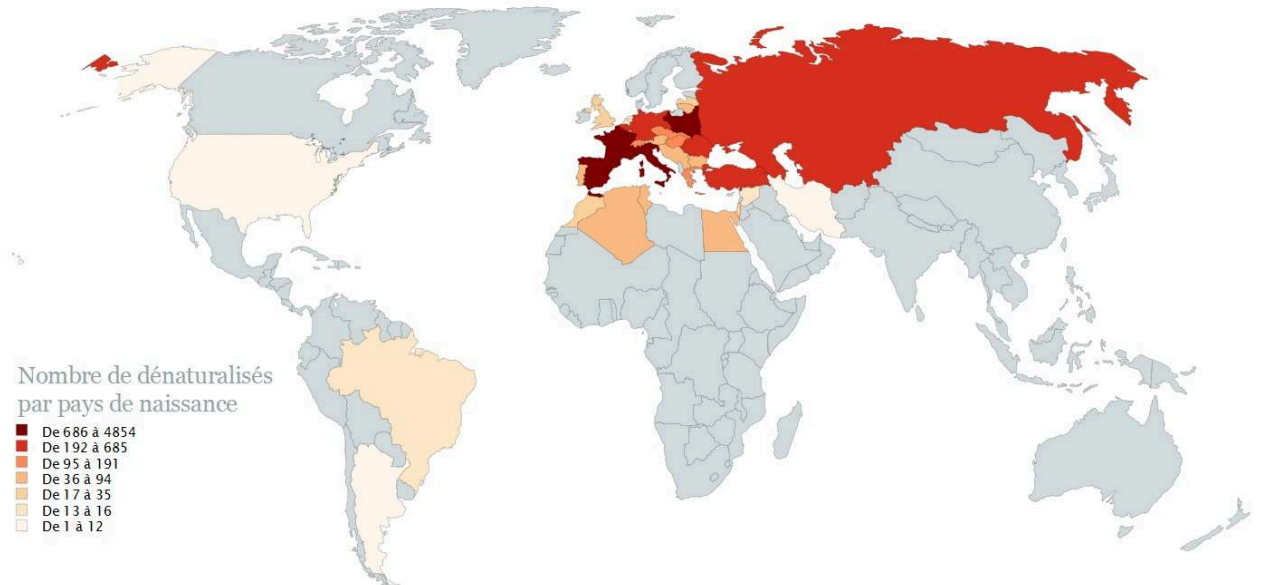

Nombre de dénaturalisés par pays de naissance (carte réalisée au moyen de https:// historicalmapchart.net/)

L'étude des originaires d'Italie et de Pologne, respectivement deuxième et troisième pays de naissance les plus représentés parmi les dénaturalisés, est singulièrement pertinente : les dénaturalisations touchent plus particulièrement ces nationalités pour des raisons différentes. Si les Italiens sont très représentés parmi les dénaturalisés, c'est qu'ils le sont également parmi les naturalisés. En revanche, les pays d'Europe de l'Est ou « orientaux » sont tous surreprésentés. Les Polonais, par exemple, sont deux fois plus nombreux à faire l'objet d'un retrait que ce qu'impliquerait leur proportion 
dans les naturalisés d'après 1927. Avec les Roumains, la proportion est multipliée par 4. Pour les pays considérés par la commission comme des pays " orientaux ", la Grèce et la Turquie, c'est respectivement 3 et 2,5 fois plus de dénaturalisés que leur proportion dans la population des naturalisés.

\section{Proportions de naturalisés et de retrayés par pays}

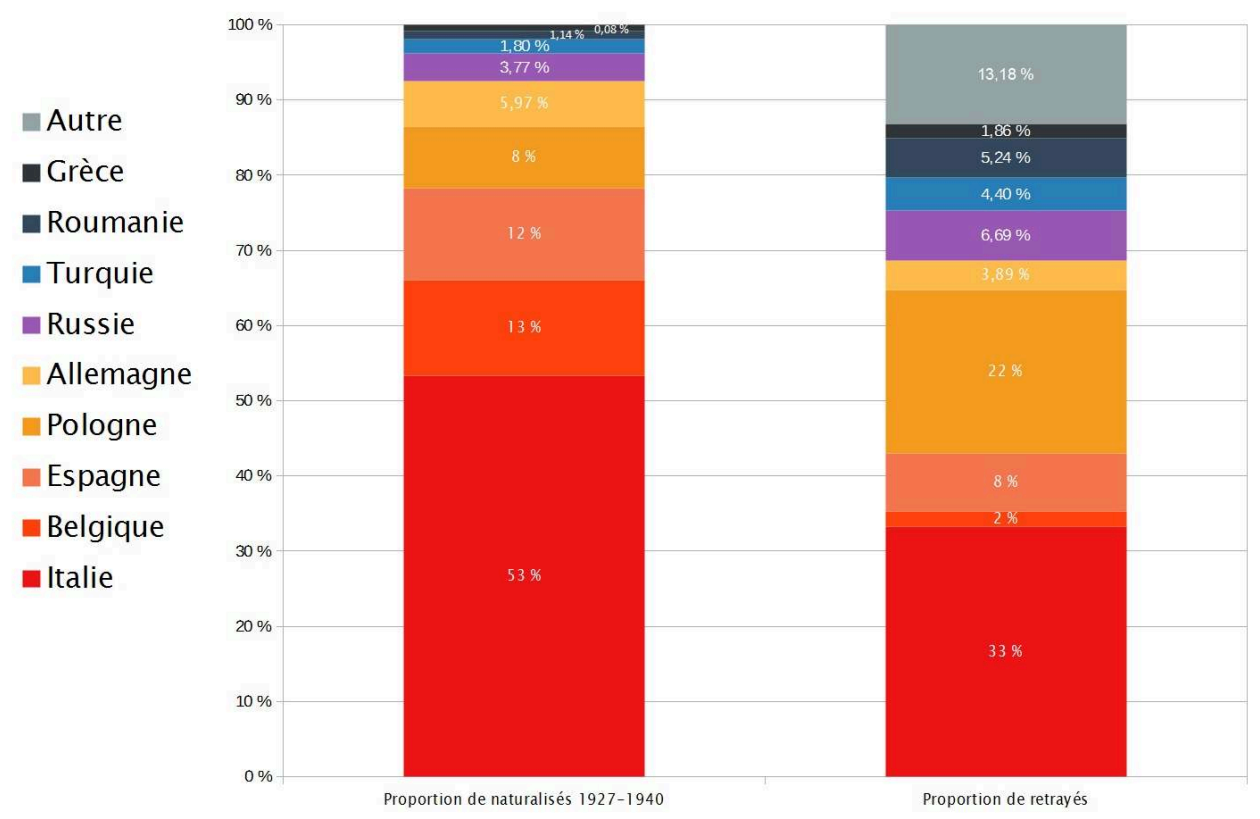

Proportions de naturalisés et de retrayés par pays

En effet, la commission de révision a d'emblée réparti les naturalisés entre «acceptables » et «indésirables ». Dans la première catégorie, les originaires de pays voisins et de " culture ou de civilisation analogue » tels l'Italie, la Belgique, l'Espagne... ceux dont les métiers exercés " comblent les déficits de main d'œuvre et l'exode des Français dans les villes » voient la naturalisation maintenue à moins de «fraudes, de condamnations graves, d'immoralité, d'agitation politique grave». Les travailleurs recrutés officiellement par contrats mais originaires de pays « sensiblement différents de la France pour la culture générale, les mœurs, les coutumes » sont a priori maintenus après une enquête " sérieuse », tout au moins « les éléments sains de cette catégorie ». Pour les naturalisés qualifiés par le président de la commission Jean-Marie Roussel de " réfugiés dont la mentalité et les habitudes sont très éloignées des nôtres », originaires d'Europe de l'Est ou "même » de pays orientaux (Syrie, Liban...), la commission s'est montrée beaucoup "plus stricte dans l'examen des titres ». Elle a notamment rejeté ceux qui exerçaient des métiers jugés encombrés "ouvriers tailleurs, coiffeurs » ou «dangereux : brocanteurs, forains, tenanciers de bar... ». Il s'avère, constate Roussel, que beaucoup de ces naturalisés étaient juifs. Il assure que la commission a abordé cette "délicate question», «sans parti-pris et sans passion, sans préjugé racial ou confessionnel » prenant même des " précautions » pour ne pas aggraver la situation de ces "persécutés ". C'est après la guerre, dans un mémoire en défense lors de son épuration administrative, que Roussel écrit ces quelques lignes. La base Dénat et les dossiers de naturalisation permettent de contrebalancer ces affirmations en montrant notamment qu'il y a un avant et un après 1943 dans le fonctionnement de la 
commission. Une extrême sévérité et des commentaires lapidaires dans les dossiers des premiers dénaturalisés ( «sans intérêt national ", par exemple) ${ }^{10}$, davantage d'enquêtes et d'atermoiements à partir de 1943. C'est évidemment dans la dernière catégorie de naturalisés évoqués par Roussel que se trouvent les victimes «absolues» de la dénaturalisation : les personnes déportées parce que dénaturalisées.

\section{Les Juifs sont-ils surreprésentés parmi les dénaturalisés?}

Pour tenter de répondre à cette question, le prérequis est de connaître la proportion de Juifs naturalisés après 1927 pour la comparer à celle des dénaturalisés de 1940-1944, comme il est facile de le faire avec les nationalités d'origine. En août 1943, le sousdirecteur du Sceau avait été sommé de répondre dans la journée à une rafale de questions sur: le nombre de Juifs naturalisés depuis 1927; le nombre de Juifs dénaturalisés; le nombre de Juifs maintenus par la commission; le nombre de Juifs dont la situation n'a pas encore été examinée. Dans sa note du 26 août 1943, il affirme ne pas pouvoir connaitre le nombre de Juifs naturalisés depuis 1927, les postulants n'ayant pas été interrogés sur leur religion lors de la procédure de naturalisation. Le sous-directeur répond toutefois dans l'urgence : 7053 juifs auraient été dénaturalisés à la date du 26 août 1943. Mais Ferdinand de Brinon, délégué général du gouvernement dans les territoires occupés, donne un autre chiffre dans un télégramme daté du 2 août 1943 adressé à Laval : 16508 dossiers auraient été «retenus» à ce jour par la commission, dont 6307 dossiers de Juifs (un chiffre jugé dérisoire par ce même Ferdinand de Brinon). Il affirme qu'un tiers des 539280 dossiers déjà examinés concerneraient des Juifs. Tous ces chiffres, instrumentalisés par les acteurs de l'époque, sont donnés dans un contexte de tension avec les autorités d'occupation (et le commissariat général aux questions juives) qui veulent mettre en place une nouvelle loi qui dénaturaliserait tous les Juifs naturalisés depuis $1927^{11}$. C'est ici que la base Dénat peut enrichir le débat en montrant que les originaires des pays où la population juive est très représentée ont été plus particulièrement touchés par la dénaturalisation. Toutefois le seul moyen de connaître le nombre de Juifs parmi les dénaturalisés de Vichy est de reprendre les dossiers correspondant aux 15154 personnes figurant dans le fichier, d'ouvrir ces dossiers, de retrouver la mention "Juifs" ajoutée en haut à droite de l'avis de retrait, de supposer qu'il s'agit effectivement de dénaturalisés juifs, de vérifier que le décret de retrait, sans doute pour repérer et compter facilement le nombre de Juifs dénaturalisés, de supposer qu'il s'agit effectivement de dénaturalisés juifs, de vérifier que le décret de retrait concerne toute la famille et de compter les membres de cette famille. La base Dénat rend un tel travail, sinon facile, du moins possible.

Cependant, par des approches intuitives et en considérant que le bureau du Sceau a donné des chiffres à peu près exacts, il semble que l'on puisse raisonnablement établir que 30 à $40 \%$ du total des dénaturalisés de la loi du 22 juillet 1940 sont juifs. Mais, pour prendre l'exacte mesure de ce pourcentage, encore une fois, il manque essentiellement le chiffre des naturalisés juifs entre 1927 et 1940.

28 La question posée par la dénaturalisation des Juifs reste celle des conséquences d'une mesure qui les place dans un statut d'étranger. Quelle est notamment l'incidence des avis de la commission de révision sur la déportation des personnes privées de la 
nationalité française ? Bernard Laguerre, dans son article "Les dénaturalisés de Vichy " ${ }^{12}$, regrettait que l'on ne puisse aisément comparer les noms des 15154 dénaturalisés contenus dans les décrets d'une part et la liste des déportés par convoi établie par Serge Klarsfeld d'autre part. En effet, au moment où Bernard Laguerre écrit son article (1988), aucune des deux listes à comparer n'est classée par ordre alphabétique. C'est pourquoi l'auteur avait dû se résoudre à étudier une communauté réduite, celle des 183 Juifs de Salonique repérés dans les décrets. Aujourd'hui, grâce à la saisie du fichier et au partenariat des Archives nationales avec le Mémorial de la Shoah, cet exercice est possible. Les bases respectives des deux institutions ont été comparées et même échangées. Mais ce qui apparaissait comme une opération relativement simple s'est avéré complexe : un premier tri sur les noms de famille donne 700 noms de personnes à la fois dénaturalisées et déportées. En effet, les noms diffèrent parfois d'une seule lettre, rendant la comparaison inopérante, les prénoms (francisés ou non) sont à considérer avec les plus grandes précautions. Quant aux dates de naissance, elles peuvent être imprécises dans la base des victimes de la Shoah en France et très précises dans la base Dénat. D'autres tris par date de naissance et ville de naissances, des croisements de données à la fois larges puis très précis ont été effectués. Aujourd'hui, 902 personnes ont été identifiées comme dénaturalisées et déportées. Mais il est probable que ce chiffre inférieur à toutes les projections établies jusqu'alors soit appelé à évoluer.

Parmi ces 902 personnes figurent les victimes absolues de la dénaturalisation. Si l'on part du postulat qu'à partir de fin 1942-début 1943, Juifs français ou Juifs étrangers sont déportés sans distinction, il ne faut considérer que les personnes déportées avant cette période. Si l'on fait un tri par convoi et que l'on ne retient que les personnes dénaturalisées et déportées avant le convoi 48 (13 février 1943), on obtient le total de 405 noms dont on pourrait affirmer qu'il s'agit des victimes absolues de la dénaturalisation. Mais il faut encore nuancer les chiffres : des décrets de retraits sont par exemple pris après la déportation : la dénaturalisation n'aurait donc, a priori, pas eu d'incidence sur cette déportation. Par ailleurs, des Juifs français ont été déportés avant le convoi 48. C'est donc un travail d'étude au cas par cas, dossier par dossier, qu'il faut entreprendre.

Que nous apprennent la base Dénat et les dossiers de naturalisation sur les victimes absolues du retrait de la nationalité ? Grâce aux interrogations croisées s'esquissent quelques traits: ce sont des naturalisés récents (tri par date de naturalisation), des retrayés de la première heure de la fin 1940 et du début 1941 (tri par date de retrait). Parmi eux, les juifs de Salonique sont précocement dénaturalisés et précocement déportés (tris par pays/ville d'origine, par date de retrait et par convoi). La consultation des dossiers de naturalisation montre que des professions comme médecins, avocats, banquiers sont très représentées. Le recours aux dossiers permet d'ailleurs de dresser un portrait plus précis et surtout plus incarné, car au-delà des chiffres, il y a des vies bouleversées, parfois détruites.

31 Marie Ajchenbaum ${ }^{13}$ est une victime absolue de la dénaturalisation. Sa famille a été dénaturalisée par le premier décret du $1^{\mathrm{er}}$ novembre 1940. Ses deux frères ont vu leur recours accepté pour faits de guerre mais cette décision ne s'applique ni à leurs parents ni à leur sœur Marie. Une lettre désespérée d'Aaron (Henri), le frère de Marie, réclamant qu'elle bénéficie elle aussi de l'annulation de la dénaturalisation figure dans le dossier de naturalisation: "Ma sœur [...] a été arrêtée le 16 juillet 1942 [...] et 
incarcérée dans le camp de Drancy [...] Ceci en attendant sa déportation prochaine pour une destination inconnue. J'ai appris que ce jour, seuls les éléments étrangers furent arrêtés. Donc ma sœur arrêtée comme telle n'est plus considérée comme française par les autorités d'occupation, quelques personnes de nationalité française, arrêtées par erreur furent relâchées après vérification d'identité et justification de la qualité de Français [...] J'ai l'honneur de vous demander de bien vouloir rapporter d'urgence cette mesure en ce qui concerne ma sœur qu'il y a encore possibilité de sauver de la déportation. ». Alors que son frère écrit cette lettre le 17 août 1942, Marie a déjà été déportée par le convoi 12 du 27 juillet 1942.

Toutefois, avec la famille Gattegno ${ }^{14}$, famille juive de Salonique, les limites d'une étude uniquement fondée sur les chiffres apparaissent à l'évidence. Cette famille est composée de Bienvenida et Moise, les parents, d'éléonore et Élie, les deux enfants mineurs, et d'Alice, la fille aînée, naturalisée individuellement. Tous, sauf Alice, sont dénaturalisés en juin 1941; leur recours est rejeté en août 1942 et toute la famille, y compris Alice, est déportée par le convoi 36 du 23 septembre 1942. Or lorsque l'on observe la liste de ce convoi, on s'aperçoit qu'Alice, qui a conservé la nationalité française, est inscrite sous cette nationalité alors que sa sœur Éléonore, dénaturalisée, l'est sous la nationalité grecque. Elles sont toutes deux déportées par le même convoi. La perte de nationalité ne semble donc pas avoir été décisive Cependant Alice est arrêtée au même domicile que sa famille dénaturalisée. Cette circonstance a-t-elle décidé de son sort?

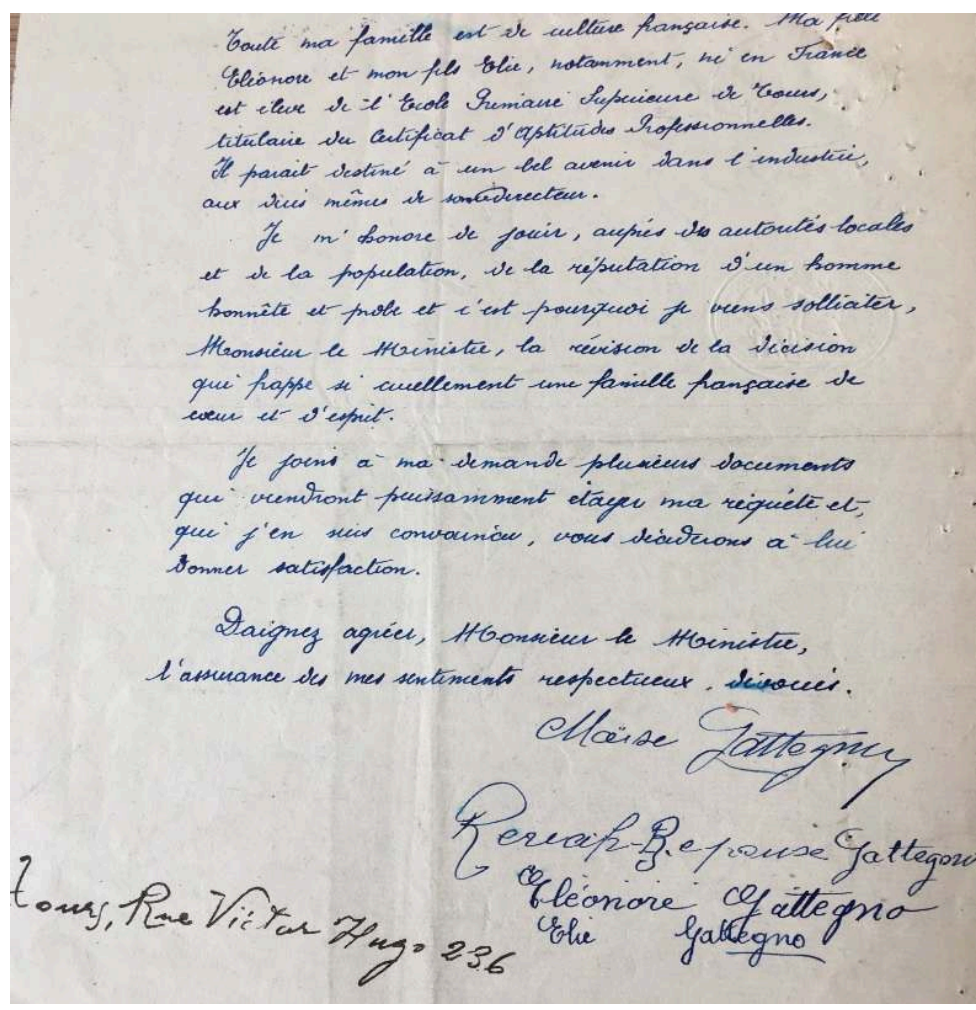

Recours gracieux présenté par la famille Gattegno ${ }^{15}$

Dès le 8 octobre 1941, un avocat dépose à l'intention de la commission de révision un mémoire dans l'intérêt des frères Goldenberg ${ }^{16}$, dénaturalisés en juin 1941. Il y expose les dangers encourus par les Juifs privés de la nationalité française. Ce n'est pourtant que deux ans plus tard, dans une note du 24 septembre 1943, que Jean Trannoy, 
substitut chargé de la direction des services du secrétariat de la commission de révision des naturalisations, écrit : "Monsieur le Président de la Commission de Révision des Naturalisations, a fait savoir, à la séance du 21 septembre [1943], que désormais, en ce qui concerne les Juifs, la Commission ne proposerait la mise aux décrets de retrait qu’à l'encontre des intéressés dont on ignore le domicile actuel ». Jean Trannoy préconise également de s'abstenir de mentionner la dernière adresse connue "si l'on veut, comme le désire la Commission, éviter que les intéressés puissent être retrouvés " ${ }^{17}$. Dans les décrets originaux de 1943 et 1944 conservés aux Archives nationales ${ }^{18}$ la formule suivante est effectivement fréquemment employée : "ayant demeuré à » en lieu et place de "demeurant à ", répandue auparavant. C'est pourquoi un champ intitulé «SDC» a été ajouté à la grille de saisie de la base Dénat dans le but de déterminer la proportion de ces "sans domicile connu " parmi les dénaturalisés mais l'instrument de recherche est perfectible en ce domaine, car ce champ, requérant une forme d'interprétation des éléments inscrits sur la fiche, est complété de manière trop aléatoire par les annotateurs.

En 1943, la commission de révision prend donc des décisions bien tardives qui montrent qu'elle semble avoir (enfin) conscience des dangers que les retraits de nationalité et leur notification font courir aux Juifs.

Le cas de la famille Angel ${ }^{19}$ illustre à les difficultés d'identification parfois rencontrées et l'aspect mémoriel du projet, déjà évoqué. La famille Angel figure sous ce nom dans la base des victimes de la Shoah en France et sous le nom Salomon dans la base Dénat. Comment rapprocher ces noms dans deux bases différentes? C'est le tri par ville de naissance des enfants (en l'occurrence, Lille) qui a permis à la faveur de nombreuses relectures, cette identification particulièrement complexe. Lors de la naturalisation, le prénom Salomon a été inscrit comme nom de famille et les actes de naissance des enfants portent ce nom. Les parents et les sept enfants ont été déportés, dans les convois 8 pour le père Salomon, ses enfants Esther et Isaac, et le convoi 34 pour Léa, la mère, et les enfants, Rachel, Joseph, Sarah, Jacques et Yvonne. Grâce à la base, on identifie formellement Léa Angel, qui porte parfois les prénoms de Louise ou Lucie, et la petite Yvonne née le 23 février 1940, inscrite puis rayée sur la liste du convoi 8 sous le prénom Iwan. Une note du préfet de Loire-Atlantique conservée dans le dossier de naturalisation informe le garde des Sceaux que le décret de retrait a été affiché en préfecture et publié dans le journal Le phare le 24 septembre 1942, un mois après la disparition de la famille à Auschwitz. Il semblait de première importance que la population locale soit bien informée que la petite Yvonne Angel, 2 ans née à SaintNazaire, ses parents, ses six frères et sœurs âgées de 5 à 17 ans, aient été jugés « indignes de conserver la nationalité française ». 


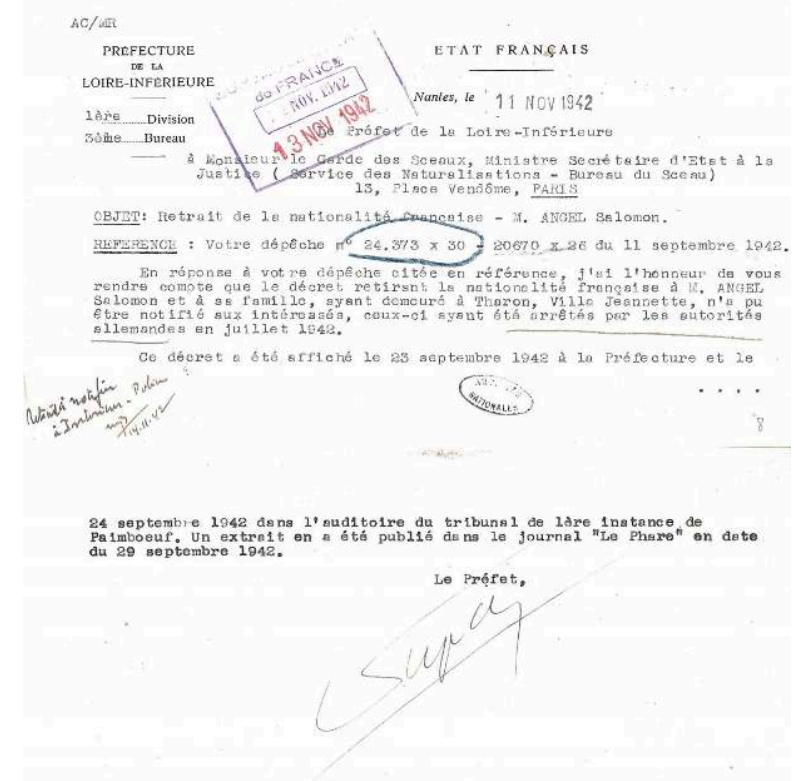

Notification du retrait de la nationalité française de la famille Angel/Salomon

Les lettres de recours gracieux dans lesquelles les familles énumèrent les conséquences de la perte de la nationalité française témoignent du traumatisme du rejet hors de la communauté nationale. S'agissant des Juifs, leur vie peut être mise en danger. Les victimes « absolues » de la dénaturalisation existent bel et bien. Mais les seuls chiffres, paraissant pourtant clairs au premier abord, ne peuvent être une réponse. Il est très difficile de déterminer quelle est la part de la dénaturalisation dans le sort de tels ou tels dénaturalisés/déportés. Pour certains elle fut décisive, pour d'autres elle fut un élément de plus dans la perte de tous les droits, pour d'autres encore la procédure, les enquêtes, les rapports, les signalements ont pu fragiliser la fuite ou le refuge. Les dossiers de naturalisation peuvent apporter des réponses et les échanges d'informations avec le Mémorial de la Shoah sont certainement essentiels.

Le nouvel outil présenté ici ne permet pas de répondre à toutes les questions : il ouvre des pistes. C'est aux chercheurs, aux familles à l'utiliser maintenant. D'autres victimes «absolues » sont sans doute à identifier. Nous avons été heureux d'avoir pu, en quelque sorte, accéder au vœu de Bernard Laguerre dans son article de 1988 : comparer les listes des dénaturalisés et des déportés et ainsi mieux connaître les victimes, notamment les victimes absolues des dénaturalisations opérées par Vichy entre 1940 et 1944. 


\section{BIBLIOGRAPHIE}

LAGUERRE Bernard, «Les dénaturalisés de Vichy. 1940-1944 ", Vingtième siècle. Revue d'histoire, 20, 1988, p. 3-15.

POINSOT Annie, «Retrait, maintien, enquête ». La commission de révision des naturalisations (1940-1944): un instrument de la politique xénophobe et antisémite de Vichy?, mémoire de master 2, université Paris I Panthéon - Sorbonne, octobre 2013.

ZALC Claire, Dénaturalisés. Les retraits de nationalité sous Vichy, Paris, Seuil, 2016.

\section{NOTES}

1. Décret-loi du 22 juillet 1940 portant sur la révision des naturalisations, Journal officiel du 23 juillet 1940, p. 4567.

2. SCHOR Ralph, L'opinion française et les étrangers (1919-1939), Paris, Publications de la Sorbonne, 1985.

3. Archives nationales, $3 \mathrm{~W} / 46$ Haute Cour de justice, instruction Alibert.

4. Archives nationales, A/1893 à 1895 («État français : décrets individuels classés par objet », 1942-1944).

5. Circulaire du ministère de la Justice du 3 mai 1941 donnant les instructions aux préfets sur la procédure de notification du retrait de la nationalité française et à laquelle il est fait référence dans les dossiers de naturalisation.

6. Décret-loi du 22 juillet 1940 portant sur la révision des naturalisations, Journal officiel du 23 juillet 1940

7. Archives nationales, BB/27/1422 à 1445 , fichier des retraits de la nationalité française.

8. Archives nationales, $3 \mathrm{~W} / 46$ Haute Cour de justice, instruction Alibert.

9. Mémoire en défense présenté par J.-M. Roussel lors de son épuration administrative, Archives nationales, $\mathrm{BB} / 30 / 1840$.

10. Archives nationales, $19770890 / 73$, dossier $27328 \times 36$.

11. Archives nationales, $19960100 / 1$.

12. «Les dénaturalisés de Vichy. 1940-1944 », Vingtième siècle. Revue d'histoire, 20, 1988, p. 3-15.

13. Archives nationales, BB/11/10663, dossier 47410 X 28 : famille Ajchenbaum.

14. Archives nationales, $19770890 / 96$, dossier 28596 X 36 : famille Gattegno.

15. Archives nationales, $19770890 / 96$, dossier 28596 X 36 : famille Gattegno.

16. Archives nationales, $19770890 / 73$, dossier 27326 X 36 : famille Goldenberg.

17. Archives nationales, 19960100/1 («Réglementation sur la naturalisation », 1940-1944).

18. Archives nationales, A/1894 à 1895 ("État français : décrets individuels classés par objet », janvier 1943-août 1944).

19. Archives nationales, BB/11/13308, dossier 24373 X 30 : famille Angel. 


\section{AUTEURS}

\section{ANNIE POINSOT}

Chargée des fonds liés à la nationalité, département de la Justice et de l'Intérieur, direction des fonds, Archives nationales

\section{BERNARD RAQUIN}

Administrateur documentaire, département de la maîtrise d'ouvrage du système informatique, direction de l'appui scientifique et technique, Archives nationales 


\title{
Rôle du préfet, de ses services et des maires dans l'instruction des dossiers de dénaturalisation : l'exemple de la Meurthe-et-Moselle
}

\author{
Jean-Claude Magrinelli
}

\begin{abstract}
Ce média ne peut être affiché ici. Veuillez vous reporter à l'édition en ligne http:// books.openedition.org/pan/1146
\end{abstract}

2 C'est à l'occasion d'un travail de recherche sur les ouvriers de Lorraine dans la période 1936-1946 que l'attitude envers les travailleurs étrangers, nombreux dans cette région, des trois gouvernements successifs, celui de la III ${ }^{e}$ République finissante puis celui du régime de Vichy qui collabore avec l'occupant, enfin celui de la IV République naissante, devait être interrogée.

La Meurthe-et-Moselle est en effet, malgré la crise des années 30, le premier producteur de fonte et d'acier du pays, avec ses 42 mines et 21 usines sidérurgiques et métallurgiques qui travaillent pour la Défense nationale. C'est un département où le prolétariat industriel est majoritaire dans la population active (12000 mineurs de fer, 40000 métallurgistes, 6000 ouvriers dans l'industrie du sel, 20000 dans l'industrie textile et du cuir). $12 \%$ des habitants $(67000)$ du département sont étrangers, aux deux tiers Italiens et le reste Polonais. C'est un département stratégique pour l'occupant, puisque les voies de communication routières, ferrées, fluviales, téléphoniques entre Paris et l'Allemagne y passent. C'est enfin un département où les organisations ouvrières, politiques et syndicales sont devenues durablement puissantes avec l'avènement du Front populaire, gagnant la majorité des ouvriers à l'antifascisme. Fin 1937, le département compte près de 7000 adhérents à la SFIO, 4300 adhérents au Parti communiste et 1000 jeunes communistes - c'est le seul parti à avoir constitué des cellules d'entreprises -; près de 91000 syndiqués à la CGT (un salarié sur deux). L'émergence et la permanence sur le devant de la scène politique et sociale d'une force 
ouvrière unifiée, organisée, combative, antifasciste, sont devenues depuis cette époque une tendance lourde de l'histoire régionale. Cette force posait en soi la question de la place de la classe ouvrière dans la société, mais aussi celle de la place des ouvriers immigrés, dont la participation au Front populaire a été décisive en Meurthe-etMoselle.

4 L'échec de la grève du 30 novembre 1938 (8 000 grévistes dans l'industrie soit $12 \%$ ), qui a donné lieu à une répression patronale d'ampleur (198 licenciés et 118 sanctionnés), traduit la modification du rapport des forces en faveur des conservateurs. Le gouvernement Daladier, par décrets-lois, entend « remettre la France au travail » et en terminer avec « la semaine des deux dimanches ", c'est-à-dire avec les 40 heures. C'est dans ce contexte de réaction sociale que de premières mesures visant les étrangers sont prises. Au motif de tensions internationales, ils deviennent pour le gouvernement de possibles éléments de troubles intérieurs, plus généralement des suspects, surtout s'ils sont venus grossir les rangs de la CGT, du PCF, de l'Union populaire italienne et ont participé aux grèves, aux actions de soutien à la République espagnole sous le Front populaire. Il est remarquable que les brigadistes italiens et polonais revenus d'Espagne aient été systématiquement surveillés en Meurthe-et-Moselle à l'occasion de la grève générale du 30 novembre 1938. La politique de dénaturalisation de Vichy s'inscrit donc dans un long processus, commencé fin 1938 avec les décrets-lois Daladier sur les étrangers.

5 Pendant la "drôle de guerre ", l'étranger suspect est particulièrement visé. En application des instructions ministérielles du 17 septembre 1939, le préfet Léon Bosney demande à la gendarmerie, par circulaire du 30 septembre, de lui fournir la liste « des étrangers ou étrangères indésirables, suspects au point de vue national ou dangereux pour l'ordre public, en vue de leur internement éventuel, se trouvant actuellement encore dans votre circonscription ou votre secteur ", avec rapport sur chacun d'eux. Cela signifie que le service des étrangers de la préfecture instruit désormais des dossiers individuels de nature politique, alimentés par les brigades de gendarmerie. Cette mission s'ajoute à ses missions administratives traditionnelles de délivrance des cartes d'identité ou de séjour. Cela signifie donc aussi que la préfecture tient un fichier des suspects étrangers.

6 Avec l'Occupation et l'installation à Vichy du gouvernement Pétain, un pas supplémentaire est franchi. Ce dernier entend, sinon débarrasser le pays des étrangers indésirables, du moins les placer sous contrôle étroit et, pour les plus dangereux, les priver de la citoyenneté française qu'ils auraient pu acquérir. L'anticommunisme va de pair ici avec la xénophobie. Voici les mesures prises entre juillet et décembre qui complètent celles prises depuis l'automne 1938 :

- 22 juillet 1940 : loi portant sur la révision des naturalisations intervenues depuis la loi du 10 août 1927 ;

- 3 septembre 1940 : loi relative aux mesures à prendre à l'égard des individus dangereux ; elle prolonge les dispositions du décret-loi du 18 novembre 1939 ;

- 27 septembre 1940 : loi portant création des Groupements de travailleurs étrangers ;

- 10 octobre 1940 : circulaire relative aux étrangers indésirables et en surnombre dans l'économie nationale ;

- 23 décembre 1940 : loi donnant plein pouvoir aux préfets en matière de mesures à prendre à l'encontre des étrangers indésirables. 
7 Analysons comment cette politique a été appliquée en Meurthe-et-Moselle par les autorités préfectorales pendant l'Occupation. Ce sont les critères politiques, plus que tout autre, qui ont été retenus contre les étrangers, les mesures prises visant en priorité des militants ouvriers.

8 La première mesure prise est une circulaire $\mathrm{n}^{\circ}$ 71/D d'août 1940, adressée aux maires et commissaires de police, concernant la régularisation de la situation des étrangers par la délivrance de nouvelles cartes d'étranger après les " événements qui se sont produits depuis juin dernier ", c'est-à-dire la débâcle. Il est demandé une liste comprenant les nom, prénoms, date et lieu de naissance, profession et nationalité de tous les étrangers de la commune, avec une rubrique «indésirables». Les maires transmettent à partir du 4 septembre 1940 les listes d'étrangers de leurs communes. Par exemple, la liste d'Auboué compte 1381 noms ; celle d'Homécourt, 2567 ; Dombasle, 118; Jarny, 861 ; Lunéville, 417; Varangéville, 249. Seuls trois maires (Malzéville, Lunéville et Jarny) renseignent la rubrique "étrangers indésirables ", fournissant en tout et pour tout des renseignements sur deux familles. ${ }^{1}$ Pour tous les autres, il n'y a pas d'« étrangers indésirables" dans leur commune. Dès la fin de septembre 1940, le service des étrangers de la $1^{\text {re }}$ division de la préfecture commence donc à reconstituer et à réactualiser, compte tenu de l'exode des populations en juin 1940, le fichier de tous les étrangers résidant dans le département. Les résultats de ce premier recensement témoignent qu'il subsiste encore, chez la plupart des maires de Meurthe-et-Moselle, élus démocratiquement aux élections municipales de 1935, une conscience républicaine.

9 En novembre 1940, nouveau recensement. Par circulaire $\mathrm{n}^{\circ}$ 110/D du 11 novembre 1940, " conformément aux instructions des autorités supérieures françaises", le préfet demande aux maires ainsi qu'aux commissaires de police de lui fournir :

- un état numérique, par nationalité, des étrangers résidant dans leur commune ;

- la liste nominative, par nationalité, des « étrangers indésirables » (communistes, expulsés et repris de justice) ;

- la liste nominative des étrangers indigents, par nationalité ;

- la liste nominative spéciale des réfugiés espagnols expulsés, indésirables, indigents ou hébergés au compte de l'État en indiquant ceux qui veulent et peuvent être rapatriés d'une part, ceux qui ne veulent ou ne peuvent être rapatriés d'autre part ;

- l'état numérique des réfugiés espagnols orphelins ;

- l'état des effectifs des compagnies de travailleurs qui pourraient exister dans leur commune. Tableau des « indésirables » (novembre 1940)

\begin{tabular}{|l|l|l|l|l|l|}
\hline Ville & Communistes & $\begin{array}{l}\text { Expulsés } \\
\text { refoulés }\end{array}$ & Indigents & Autres motifs & Totaux \\
\hline Auboué & 12 & 3 & - & - & 15 \\
\hline Crusnes & - & - & 9 & - & 9 \\
\hline Dombasle & 1 & - & - & - & 1 \\
\hline Giraumont & - & - & 8 & - & 8 \\
\hline Herserange & 1 & - & - & - & 1 \\
\hline
\end{tabular}




\begin{tabular}{|l|l|l|l|l|l|}
\hline Jarny & - & 3 & - & - & 3 \\
\hline Joeuf & - & 6 & - & - & 6 \\
\hline Lunéville & 1 & 1 & - & - & 2 \\
\hline Malzéville & 1 & - & - & - & 1 \\
\hline Moutiers & 31 & 1 & - & 15 & 47 \\
\hline Nancy & 5 & 2 & 4 & 7 & 18 \\
\hline Pont-à-Mousson & - & 1 & 2 & - & 3 \\
\hline Toul & 1 & - & - & 1 & 2 \\
\hline Trieux & 4 & - & - & - & 4 \\
\hline Totaux & 57 & $\mathbf{1 7}$ & $\mathbf{2 3}$ & $\mathbf{2 3}$ & $\mathbf{1 2 0}$ \\
\hline
\end{tabular}

Ce second recensement est important par la notion d'« indésirables » présentée dans la rubrique $\mathrm{n}^{\circ} 2$ de l'enquête. Sont qualifiés ainsi les communistes, les expulsés et refoulés en sursis, les pauvres et autres (non spécifiés). La plupart des villes industrielles à forte concentration ouvrière et étrangère fournissent un "état néant" d'indésirables. 14 villes ont recensé 120 « indésirables ». La moitié des "indésirables» sont classés " communistes ». Les villes d'Auboué, Moutiers et Nancy se distinguent par le nombre élevé d'« indésirables communistes » ( $84 \%$ du total). Les municipalités d'Auboué et Moutiers, élues en 1935 et dirigées par des hommes de confiance des directions d'usine ou de mine, mentionnent souvent «meneur de grèves » ou «s'est fait remarquer dans les grèves ». Figurent dans la liste d'Auboué : Gino Parentelli, Mario Tinelli, Davide Foggi, Cleto Gervasi, Diulio Imolesi, Alfredo Pagnoni, des militants particulièrement visés par la répression en 1942. Nancy mentionne Abraham Balbin qui sera arrêté comme "juif étranger ». Dans la série "Autres motifs»sont mentionnées des personnes aussi diversement appréciées comme " de mauvaises mœurs », « ivrognes ", " voleurs ", « trafiquants ", " paresseux ». C'est à Moutiers et à Nancy que le nombre de personnes classées dans cette catégorie est le plus important. 14 maires, sur les 599 communes que compte la Meurthe-et-Moselle, ont répondu partiellement à cette partie de l'enquête, soit $2 \%$. La fibre républicaine reste encore agissante chez la plupart d'entre eux. ${ }^{2}$ Une relance est faite par une circulaire du préfet du 6 janvier 1941, puis une autre le 10 mars $1941 .^{3}$

11 La caractéristique du processus de recensement des « étrangers indésirables » est qu'il implique la responsabilité des maires. En d'autres termes, ces derniers participent désormais, par les listes qu'ils constituent et les appréciations qu'ils portent, à la préparation des mesures de répression contre les étrangers. En la matière, ils apportent au préfet les informations complémentaires aux informations fournies par les services de gendarmerie et de police.

Une mention particulière doit être faite pour les Israélites étrangers, parce qu'ils ont été visés à la fois par les autorités françaises et par l'occupant. La liste des juifs allemands installés dans l'arrondissement de Nancy est demandée par la 
Feldgendarmerie au cabinet du préfet courant juillet 1940. Une circulaire est adressée aux maires par le préfet, le 9 août 1940, afin qu'ils dressent la liste des résidents juifs allemands. Le 20 septembre, la Feldkommandantur 591 de Nancy (FK 591) demande cette fois au préfet la liste des ressortissants allemands résidant dans le département. Le préfet Bosney avait déjà adressé, le 29 août 1940, aux maires et commissaires de police une circulaire en ce sens. Le chef des services administratifs de la FK 591 obtiendra du nouveau préfet Jean Schmidt (en poste depuis septembre 1940), début mars 1941, un état numérique du fichier des étrangers domiciliés dans le département, apatrides et émigrants allemands compris, classés par nationalité. Les fiches d'étrangers des personnes juives sont marquées en rouge par un grand J ${ }^{4}$. Le 9 mai 1941, Jean Schmidt demande aux sous-préfets de Briey et Lunéville de lui adresser « en vue de leur internement administratif, la liste des juifs étrangers connus pour leur attitude contraire aux intérêts du pays ou qui se sont introduits illégalement en France, ou encore dont l'absence de ressources les place en surnombre dans l'économie nationale. » La réponse du sous-préfet de Briey n'est pas connue. Le sous-préfet de Lunéville adresse au préfet un rapport sur le «vieux militant » Abramowicz Lejb et écrit : « Par ailleurs, il y aurait lieu d'ajouter le nommé Rachel Abraham-Kelman, né le 14 janvier 1905 à Czestochowa, marié, père de trois enfants. Naturalisé français par décret en date du 4 mars 1931, il a accompli son service militaire à Lunéville. Cet individu a été secrétaire de la cellule de Lunéville. Le nommé Rachel, mobilisé au début des hostilités, est actuellement PG [«prisonnier de guerre »]. L'intéressé, sa femme et ses enfants ont été déchus de la nationalité française en vertu d'un décret en date du 21 mars 1941. $»^{5}$

Le recensement fait, la procédure d'internement commence. Ils sont 7 juifs étrangers dans le département, dont deux communistes et un sympathisant communiste :

- Osias Levine est né le 17 juillet 1911 en Pologne. Il est étudiant en faculté dentaire à Nancy où il demeure au 139, rue Saint-Dizier. D'après le commissaire central de Nancy, c'est un «sympathisant communiste hostile au régime actuel français; dangereux parce qu'instruit »;

-Abraham Balbin est né à Tomaszov en Pologne, le 12 mai 1909. Il est marié, ouvrier tailleur, réside au 96, rue Notre-Dame à Nancy depuis le début des années 1920. La police le considère comme « communiste ». Il s'engage dans les Brigades internationales parce que, explique-t-il à son frère André, «ceux qui ne comprennent pas que c'est aujourd'hui qu'il faut lutter contre le fascisme sont des fous $» .{ }^{6}$ Balbin est passé aux mains de la police allemande ;

- Lejb Abramowicz, dit «Léon », est né le 30 avril 1897 à Irena en Pologne. Il réside avec sa femme et ses quatre enfants à Lunéville, y exerçant le métier de forain. Il est naturalisé français par décret du 22 mai 1922. Sous le Front populaire, il est secrétaire de la cellule des forains et délégué local du Secours populaire français. C'est, selon le sous-préfet, un " partisan convaincu du régime soviétique ». Il a été interné 15 jours, par arrêté préfectoral du 15 mars 1941, à la suite d'une distribution de tracts à Lunéville. Il se trouve dans l'obligation d'abandonner son métier en application du statut des juifs instauré par Vichy. Il devient commerçant en bonneterie, pour faire vivre sa famille.

14 La dénaturalisation d'Abramowicz est l'étape suivante du processus. Elle permet le passage de l'intéressé du statut de naturalisé français au statut d'apatride ou d'étranger, autorisant ensuite son internement comme "israélite étranger » dans un camp spécial, en application de la circulaire du 28 avril 1941 du délégué du ministre de l'Intérieur. Il est, avec son épouse et sa fille aînée, dénaturalisé par décret du 21 mai 
1941. La délégation générale du gouvernement français pour les territoires occupés fait savoir au préfet, le 30 juin, « qu'il convient de faire diriger sur le camp de Pithiviers ou sur celui de Beaune-la-Rolande (Loiret) les sept israélites étrangers de votre département dont vous proposez l'internement. » Le préfet du Loiret, le 3 juillet, fait savoir au préfet de la Meurthe-et-Moselle que le délégué ministériel a décidé l'internement. Le camp de Beaune-la-Rolande pourra les recevoir. Le 21 juillet, Jean Schmidt sollicite de la FK 591 les laissez-passer pour les sept israélites, qui seront délivrés le 9 août. Mais le camp d'Écrouves, situé non loin de Nancy, venant de s'ouvrir le 12 août, les sept israélites sont, par arrêté préfectoral du 13 septembre 1941, " astreints d'y résider, jusqu'à nouvel ordre ».7

Levine et ses quatre camarades non communistes y entrent le 19 septembre. Abramowicz a fui en zone sud et Balbin est passé aux mains de la police allemande. Abramowicz et les membres de sa famille sont morts en déportation. Balbin est fusillé comme otage au Mont-Valérien le 21 février $1942 .{ }^{8}$ Ces deux communistes d'origine israélite, installés en France depuis fort longtemps, et naturalisé français dans le cas d'Abramowicz, sont réprimés d'abord par l'autorité préfectorale qui les recense, les interne et dénaturalise Abramowicz puis par l'occupant qui les extermine. Les deux appareils répressifs ont agi successivement contre cette catégorie d'indésirables, motivés tout à la fois par la xénophobie, l'antisémitisme et l'anticommunisme.

La loi du 22 juillet 1940 et sa circulaire d'application du lendemain permettent de procéder à la révision de toutes les naturalisations intervenues depuis 1927. C'est le $3^{\mathrm{e}}$ bureau du service des étrangers de la préfecture qui est chargé de constituer le dossier des personnes proposées à une mesure de dénaturalisation. Il centralise les pièces administratives et les rapports des maires, commissaires, gendarmes et renseignements généraux $[\mathrm{RG}]$, puis présente une note de synthèse au préfet préconisant la dénaturalisation ou non. L'appréciation donnée par les maires et les gendarmes est prépondérante. Les motifs retenus par l'autorité préfectorale et transmis au gouvernement sont, au mot près, ceux apportés par ces derniers. La notification de la décision ministérielle de retrait doit être signée par l'intéressé qui restitue en même temps le décret de naturalisation. Certains refusent de signer la notification présentée et engagent des recours en révision (Tokarz, Zampetti), qui sont tous rejetés. Ce sont donc les sous-préfets, les services de police et de gendarmerie, et les maires qui alimentent, par leurs listes et leurs rapports, le processus de dénaturalisation.

À titre d'illustration, le maire de Moutiers présente une liste de 16 personnes à dénaturaliser. Le préfet ordonne à la gendarmerie de procéder à une enquête sur ces personnes dont la moitié est connue pour avoir été des membres ou proches du Parti communiste. La procédure aboutit en 1941 à la dénaturalisation des membres des familles Vecchi (Joseph et Luigi), Boaretto (Basilio), Valli (Massimo). Elle est rendue publique par L'Écho de Nancy des 11 et 19 août 1941. S'ajoutent les frères Giuseppe et Henri Sandelli, par décret du 6 juin 1941, ainsi qu'Aldo Mazzi, dénaturalisé par décret du 14 octobre $1941 .{ }^{9}$

L'autorité préfectorale engage de sa propre initiative des procédures de dénaturalisation à l'encontre de militants internés administratifs auprès de l'administration centrale. Par exemple, le 17 juillet 1941, contre Amadeo Cavalli et Natale Passini, d'Homécourt. Le sous-préfet de Briey fait de même, le 4 septembre, contre Bruno Vanin et Louis Bresolin, habitant Valleroy. Le préfet, dans une lettre adressée le 17 octobre 1941 au sous-préfet de Briey écrit: "Vous m'avez transmis 
plusieurs listes de naturalisés connus comme ayant appartenu au Parti communiste ou participé à des agitations communistes avec avis de leur retirer la nationalité française. Pour me permettre d'engager la procédure habituelle, j'ai l'honneur de vous prier de me faire parvenir un rapport détaillé sur chacun des individus qui devra préciser en outre l'état civil et la situation familiale, la date du décret de naturalisation, les faits exacts aussi précis que possible motivant la proposition de retrait de la nationalité. » Ces dossiers ne sont pas conservés aux archives départementales.

1915 dossiers de dénaturalisation seulement sont conservés à Nancy. Tous motivent la demande de dénaturalisation par l'appartenance au Parti communiste ou des sympathies communistes. 5 militants seront internés à Compiègne, 3 seront déportés.

\begin{tabular}{|c|c|c|c|c|}
\hline Famille & Résidence & Profession & $\begin{array}{l}\text { Date de } \\
\text { naturalisation }\end{array}$ & Motifs invoqués \\
\hline AMARALLI & Mancieulles & $\begin{array}{l}\text { Mineur } \\
\text { cafetier }\end{array}$ & 1930 & Suspect au plan politique \\
\hline BOLOGNINI & Mancieulles & Mineur & 1933 & Secrétaire adjoint de cellule \\
\hline BALDINI & Hussigny & Ouvrier & 1937 & $\begin{array}{l}\text { Inscrit au PC ; s'est fait remarquer en } \\
1936\end{array}$ \\
\hline BRUNETTI & Blainville & Manœuvre & 1926 & Communiste \\
\hline BROGI & Auboué & Ouvrier & 1930 & Communiste \\
\hline CANTOVA & Herserange & Ouvrier & 1928 & Communiste \\
\hline CASAGRANDE & Villerupt & Ouvrier & 1929 & Communiste \\
\hline CHIOSTRI & Villerupt & Machiniste & 1937 & Secrétaire des Jeunesses communistes \\
\hline D'ALESSIO & Auboué & Ouvrier & 1936 & Assidu aux réunions communistes \\
\hline LUNGHI & Auboué & Ouvrier & 1935 & « Entraîneur passionné » \\
\hline MIGATTA & Auboué & Ouvrier & 1930 & Assidu aux réunions communistes \\
\hline TOKARZ & Auboué & Ouvrier & 1939 & Assidu aux réunions communistes \\
\hline SLEZAK & Dombasle & Ouvrier & 1935 & Famille de communistes convaincus \\
\hline VANINI & Herserange & Manœuvre & 1930 & $\begin{array}{l}\text { Communiste; condamné en janvier } \\
1940\end{array}$ \\
\hline ZAMPETTI & Auboué & Ouvrier & 1930 & Communiste \\
\hline
\end{tabular}

Les Archives nationales conservent les dossiers de 436 personnes dénaturalisées résidant en Meurthe-et-Moselle. Il paraît donc important de pouvoir alimenter, par les sources détenues aux archives départementales, la banque de données nationale. Les 
dossiers peuvent apporter des informations sur les autorités communales, policières, patronales parfois, qui ont apporté les informations à charge, le parcours individuel des personnes visées, l'instruction du dossier de demande de dénaturalisation, les motifs retenus par l'autorité préfectorale dans sa proposition, les conditions dans lesquelles a eu lieu la notification de décision ministérielle aux intéressés...

21 Mon propos final sera de souligner l'importance des fichiers constitués par Vichy, qui ont été mis pour beaucoup d'entre eux au service de la répression, française et hitlérienne. En Meurthe-et-Moselle, l'autorité préfectorale a voulu, en accord avec la FK 591 de Nancy, tarir les départs d'ouvriers en Moselle annexée, où les salaires sont en moyenne d'un tiers plus élevés à qualification égale. Avec l'aide des employeurs, une carte de travail est instaurée pour tous les salariés, français et étrangers, par arrêté préfectoral du 4 février 1941. Plus de 90000 travailleurs ont été ainsi « rivés » à leurs entreprises qu'ils ne pouvaient quitter sans accord préfectoral. C'est donc du fichier des salariés que dispose le préfet au printemps 1941. La préoccupation sécuritaire n'est pas absente, puisque, au même moment, il prend deux mesures coercitives génératrices de fichiers :

- par arrêté du 4 janvier 41, il remet en vigueur l'internement administratif de militants notoirement connus résidant dans les villes où ont eu lieu des distributions de tracts;

- le 3 février 1941, le préfet autorise le sous-préfet de Briey - dans son arrondissement, qui regroupe les bassins industriels de Longwy et Briey, vivent des milliers de mineurs et métallurgistes, dont une grande part d'Italiens et de Polonais - à fournir à la police allemande les listes de communistes qu'elle demande (« surtout s'ils sont étrangers » précise la lettre du préfet). ${ }^{10}$

22 Si la décision de dénaturalisation est du ressort du gouvernement, l'instruction du dossier est l'affaire du préfet, qui transmet à l'échelon ministériel des dossiers confectionnés par les services préfectoraux à partir de listes et rapports individuels établis par les sous-préfets, la gendarmerie, les commissaires de police et les maires. L'implication de ces derniers dans ce processus répressif est nécessaire, mais a rencontré quelque opposition chez certains d'entre eux. Pour que les élus locaux agissent selon ses directives ou, pour le moins, qu'ils en soient les exécutants consentants, le gouvernement Pétain promulguera la loi du 16 novembre 1940 relative à la réorganisation des corps municipaux. Le préfet Jean Schmidt mènera rondement cette « prise en main » des communes.

23 À l'été 1941, Jean Schmidt a fait constituer plusieurs fichiers que tient à jour son cabinet : celui des salariés attributaires de la carte de travail, celui des étrangers, celui des indésirables français et étrangers, celui des militants communistes notoirement connus. Ce bilan lui vaut, le 16 juillet 1941, d'être nommé préfet de la région de Nancy, qui regroupe les départements de la Meurthe-et-Moselle, de la Meuse et des Vosges.

C'est aussi à ce moment qu'est déclenchée l'invasion hitlérienne de l'Union soviétique et que la pression de la FK 591 de Nancy sur le préfet en matière de lutte anticommuniste augmente. Jean Schmidt fera recenser successivement «les anciens responsables départementaux » du parti clandestin, les «militants susceptibles d'être internés", "les meneurs dans leur milieu professionnel ou local», les "militants recherchés ", les «suspects de sympathie communistes », etc. regroupés en un fichier central détenu à son cabinet. Ce dernier devait avoir à ses yeux une grande importance, puisqu'il adresse le 19 octobre 1941 une circulaire aux sous-préfets, maires, services de gendarmerie et police, leur précisant que désormais il se chargera de transmettre aux 
autorités allemandes les listes de communistes qu'elles demandent. Tous ces fichiers, et celui des communistes tout spécialement, où l'on trouve beaucoup d'étrangers, sont devenus un enjeu de pouvoir. Ils vont être une pièce maitresse aux mains de la police française et de la police allemande dans la répression contre les groupes des Francstireurs et partisans [FTP] qui opèrent des sabotages d'installations industrielles et de voies de communication dans tout le département à partir du 4 février $1942^{11}$.

Note de l'éditeur : les dossiers de naturalisation des familles touchées par le retrait de nationalité sont répertoriés dans la base Dénat. Les familles mentionnées dans cette communication correspondent aux dossiers suivants :

\begin{tabular}{|c|c|c|}
\hline Nom & Numéro de dossier & Cote \\
\hline Bolognini & $31101 \times 33$ & $19770881 / 152$ \\
\hline Brogi & $4040 \times 30$ & BB/11/12901 \\
\hline Cantova & 61076 X 28 & $\mathrm{BB} / 11 / 10936$ \\
\hline D’Alessio & $419 \times 36$ & $19770888 / 108$ \\
\hline Lunghi & $17527 \times 35$ & $19770886 / 31$ \\
\hline Migatta & $39593 \times 29$ & $\mathrm{BB} / 11 / 12695$ \\
\hline Tokarz & $24624 \times 38$ & $19770896 / 64$ \\
\hline Slezak & $33276 \times 34$ & $19770884 / 160$ \\
\hline Vanini & 3919 X 30 & $\mathrm{BB} / 11 / 12899$ \\
\hline Zampetti & $23638 \times 30$ & BB/11/13293 \\
\hline
\end{tabular}

Les dossiers suivants, en revanche, n'ont pas fait l'objet d'une dénaturalisation, en dépit du dossier monté à la préfecture de Meurthe-et-Moselle : 


\section{ANNEXES}

\begin{tabular}{|l|l|l|}
\hline Nom & Numéro de dossier & Cote \\
\hline Amaralli & $39390 \times 29$ & BB $/ 11 / 12691$ \\
\hline Baldini & $29948 \times 36$ & $19770890 / 117$ \\
\hline Brunetti & $4988 \times 23$ & BB $/ 11 / 7996$ \\
\hline Casagrande & $22569 \times 29$ et $22570 \times 29$ & BB $/ 11 / 12355$ \\
\hline Chiostri & $53839 \times 36$ & $19770891 / 260$ \\
\hline
\end{tabular}

\section{NOTES}

1. Arch. dép. Meurthe-et-Moselle, $\mathrm{W} 160$ bis, article 5. Ce dossier relatif à ce premier recensement des étrangers rassemble les circulaires et enquêtes fournies par les communes.

2. Arch. dép. Meurthe-et-Moselle, W 160 bis, article 5, dossier «Recensement du 11 novembre 1940 » et W 84, article 13, dossiers "Étrangers indésirables » (enquête auprès des communes).

3. Arch. dép. Meurthe-et-Moselle, W 160 bis, article 4, recensement des 6 janvier et 10 mars 1941 : circulaire du 10 mars 1941 de la $1^{\text {re }}$ division, $3^{\mathrm{e}}$ bureau de la préfecture et réponses des villes classées par ordre alphabétique.

4. Arch. dép. Meurthe-et-Moselle, WM 1528, directive de la FK 591 au préfet, 28 février 1941 ; 2 Z 36 et 37, recensement des juifs étrangers, juillet-août 1940 ; W 84, article 13, instruction du 9 mai 1941 du préfet demandant un état des juifs étrangers indésirables et rapport du commissaire de police, chef de la sûreté de Nancy sur Abraham Balbin et Lejb Abramowicz, 29 mai 1941.

5. Arch. dép. Meurthe-et-Moselle, W 84 , article 13 : «Juifs étrangers indésirables internés » (1941), notamment l'instruction préfectorale du 9 mai et le rapport du commissaire chef de la sûreté à Nancy du 29 mai 1941 ; W 160 bis, article 7 : « Étrangers déchus de la nationalité française (1940-1945) », dossier Abramowicz; 2 Z 36 et 37 : fonds versé par la sous-préfecture de Lunéville, «Recensement des juifs étrangers», notamment le rapport du sous-préfet Marc Andréani au préfet, 29 avril 1941.

6. MULLER (Jean-Marie), Désobéir à Vichy, Nancy, Presses universitaires de Nancy, 1994. L'auteur le prénomme Samuel. 
7. Arch. dép. Meurthe-et-Moselle, W 160 bis, article 6, dossier des israélites allemands et étrangers, août 1940-avril 1943.

8. La base de données "Fusillés du Mont-Valérien» du site Mémoire des hommes du ministère des Armées le prénomme Szmul et donne comme date de naissance le 19 avril 1899 à Tomaszow.

9. Arch. dép. Meurthe-et-Moselle, W 160 bis, article 7. Le dossier concernant la ville de Moutiers contient l'échange de correspondances entre le maire, le sous-préfet de Briey et le préfet (novembre 1940-octobre 1941). Il contient les dossiers individuels des personnes proposées à la dénaturalisation.

10. L'Écho de Nancy, 12 février 1941 (carte de travail); arch. dép. Meurthe-et-Moselle, W 1304, article 13: lettre du préfet au sous-préfet de Briey, 3 février 1941; $1447 \mathrm{~W}$, article 1, dossier "Cour d'Appel de Nancy, section spéciale » concernant les époux Tonnelier, de Jarville : arrêté préfectoral du 9 janvier 1941 (internement administratif).

11. MAGRINeLLI (Jean-Claude), Ouvriers de Lorraine, 1936-1946. T. 3 : Dans la Résistance armée, Nancy, Kä̈ros, 2018.

\section{AUTEUR}

\section{JEAN-CLAUDE MAGRINELLI}

Chercheur au Centre régional et international de documentation et de recherche sur les mutations économiques, sociales, culturelles et politiques de l'Europe contemporaine [CRIDOR]. 


\title{
Les dénaturalisations dans les colonies
}

\author{
Laure Blévis et Claire Zalc
}

Ce média ne peut être affiché ici. Veuillez vous reporter à l'édition en ligne http://

2 Cette communication s'inscrit dans un travail en cours portant sur les dénaturalisations opérées par le régime de Vichy dans l'Empire colonial français, travail à la croisée de deux recherches précédentes de chacune des auteures : l'une sur les dénaturalisés de Vichy ${ }^{1}$ et l'autre sur les naturalisations de colonisés algériens ${ }^{2}$.

3 La question des dénaturalisations coloniales est un point peu exploré par l'historiographie ${ }^{3}$, qui s'est surtout intéressée à l'abrogation du décret Crémieux le 7 octobre 1940, loi de "dé-citoyennisation» des Juifs d'Algérie, qui redeviennent « indigènes israélites» de façon collective, à rebours de la logique des révisions individuelles de naturalisation de la loi du 22 juillet 1940.

4 Notre objectif dans ce travail consiste ainsi à croiser deux moments de l'histoire de France de mise en place d'un droit de la discrimination raciale (le régime de Vichy et la colonisation), à partir de l'étude d'un cas concret où les deux logiques, vichystes et coloniales, s'imbriquent. Pour ce faire, nous adoptons le parti d'étudier ces logiques à travers les pratiques concrètes des administrations aux prises avec la politique de retrait de nationalité mise en œuvre par l'État français.

Dans le cadre de cette communication, il s'agira plus précisément de montrer comment la base Dénat, mise au point par les Archives nationales, donne d'ores et déjà des informations précieuses pour explorer la question des dénaturalisations coloniales et ouvre ainsi de nouvelles pistes de recherche. 


\section{La « loi du 22 juillet 1940 » et les naturalisations dans l'Empire}

6 Pour commencer cette recherche, nous sommes parties de la « loi $»^{4}$ du 22 juillet 1940 sur la révision des naturalisations. Or, cette dernière reste muette sur la question de l'Empire.

7 En effet, celle-ci n'indique pas son territoire d'application. Pourtant les législations sur la nationalité qui l'ont précédée, plus précisément les lois de 1889 et 1927, comportaient des articles précisant qu'elles s'appliquaient à l'Algérie, à la Guadeloupe, à la Martinique et à la Réunion, excluant ainsi implicitement le reste de l'Empire colonial.

8 La composition de la commission de révision des naturalisations, instituée par cette même « loi » du 22 juillet 1940, ne nous éclaire pas davantage. Si elle comprend, lors de sa mise en place en 1940, des représentants des principaux ministères concernés par les questions de naturalisation (Affaires étrangères, Intérieur, Défense, secrétariat d'État à la Jeunesse et à la Famille), aucun représentant du ministère des Colonies n'y siège ${ }^{5}$. La question de son application dans les colonies demeure donc en suspens.

Plusieurs éléments expliquent ce silence.

10 D'une part, la situation militaire dans les colonies demeure alors tout à fait incertaine. D'autre part, pour Vichy, la question coloniale se trouve ailleurs, dans le statut particulier des Juifs d'Algérie. Ceux-ci sont l'objet d'une législation spécifique, postérieure à la « loi » sur la révision des naturalisations : la « loi » du 7 octobre 1940 qui abroge le décret Crémieux du 24 octobre $1870^{6}$. Il s'agit d'un acte collectif qui met fin à un autre acte collectif. En effet, le décret Crémieux de 1870 accordait la citoyenneté française de façon collective à tous les « indigènes israélites » de l'Algérie ${ }^{7}$. Le texte du 7 octobre les dénaturalisent ou, plutôt, les «dé-citoyennisent" collectivement. Ils redeviennent «israélites indigènes ». Aucune commission de révision n'intervient alors pour étudier individuellement les dossiers concernés par cette abrogation.

11 À partir de 1942, les choses changent : les révisions des naturalisations dans l'Empire colonial semblent en effet reconnues comme partie intégrante et spécifique des tâches de la commission de révision puisqu'un arrêté du $1^{\text {er }}$ juin 1942 prévoit qu'y siège de manière permanente un représentant du ministère des Colonies. Le premier à occuper le poste est André Chimier, né en 1901 à Djerba en Tunisie. Administrateur de la France d'outre-mer, il est rédacteur depuis 1929 au ministère des Colonies, puis sous-chef de bureau au secrétariat d'État aux Colonies. Il est remplacé à la fin de l'été 1942 par Maurice Levallois, également employé au secrétariat aux Colonies. L'absence de localisation des archives de la commission de révision ${ }^{8}$ ne permet pas de connaître le rôle exact joué par ces représentants du ministère des Colonies dans l'institution mise en place par Vichy pour mener la politique de dénaturalisation. Néanmoins, il est significatif qu'à partir de 1942, on juge nécessaire d'y admettre des hommes représentant le secrétariat d'État aux Colonies. 


\section{Les dénaturalisations dans les colonies à partir de la base Dénat} pratiques de retrait des naturalisations dans les colonies. En particulier qui sont ces dénaturalisés de l'Empire ? Plus précisément, qui sont-ils au regard de la mosaïque de populations et de statuts propres à la colonisation?

\section{Spécificités coloniales} question de la nationalité, de la citoyenneté et de la naturalisation dans l'Empire colonial français. et l'A.-É. F., la Cochinchine, Madagascar), protectorats (Tunisie, Maroc, Tonkin, Annam, Laos, Cambodge) et territoires sous mandats (Liban, Syrie, Togo, Cameroun). relativement clair : les sujets ont conservé leur nationalité d'origine. Les habitants du 
Maroc sont marocains, ceux de Tunisie tunisiens ou d'autres nationalités européennes s'ils ont émigré d'Europe.

S'ils ont immigré en métropole (ou formellement a fortiori en Algérie), la loi sur la nationalité de 1889 s'applique à leurs enfants nés sur le sol français.

Par ailleurs, les gouvernements français ont mis en place plusieurs législations pour ouvrir des procédures de naturalisation dans les protectorats: ainsi de la loi du 20 décembre 1923 pour la Tunisie, ou du décret du 29 avril 1929 pour les étrangers non marocains du Maroc.

Dans les colonies, la situation est plus complexe. L'Algérie, première colonie du deuxième empire colonial, est le premier territoire dans lequel s'est forgée une nouvelle citoyenneté coloniale exportée après dans le reste de l'Empire.

En effet, longtemps incertaine, la nationalité des colonisés de l'Algérie est précisée de façon claire et définitive par le sénatus-consulte du 14 juillet 1865 : les colonisés (musulmans ou juifs) sont français, ils ont la nationalité française mais ne sont pas citoyens. Ils conservent un statut personnel religieux et relèvent du régime répressif de l'indigénat. Juridiquement les colonisés sont donc considérés comme des « indigènes » tout en possédant la nationalité française.

En conséquence, les colonisés des territoires au statut de colonie se distinguent également des étrangers stricto sensu. De ce point de vue, ils ne sont pas concernés par la loi de 1889 sur la nationalité, qui pourtant s'applique à l'Algérie, précisément parce qu'ils sont considérés comme étant déjà Français.

28 Cependant les points de ressemblance avec le statut d'étranger demeurent dans la mesure où le sénatus-consulte de 1865 prévoit que les « indigènes » peuvent demander à jouir des droits de citoyens : ils doivent alors faire une demande individuelle d'accès au statut de citoyen français (parfois indiqué « droit de cité »). Pendant toute la période coloniale, on peut estimer à environ 6000 le nombre d'Algériens à avoir bénéficié d'une telle naturalisation.

C'est ce qu'on a appelé improprement une naturalisation alors qu'on devrait plutôt la qualifier de « citoyennisation ». Cependant, cette dénomination abusive était répandue pendant toute la colonisation; en outre, les dossiers de procédure ne sont pas classés à part et se mélangent, dans les archives du ministère de la Justice, avec les dossiers de naturalisations déposées par des étrangers. Ils portent le même type de numéro (en X) et semblent traités par les mêmes services, autrement dit le bureau du Sceau. On retrouve donc aux Archives nationales ces dossiers disséminés dans les cartons d'archives de ce bureau du Sceau, qui comprennent, dans une proportion tout à fait considérable, des dossiers de naturalisation.

30 La logique du sénatus-consulte de 1865 (en particulier la procédure de naturalisation) a ensuite été exportée d'Algérie dans les différentes colonies, étayée par un ensemble de textes juridiques créant des procédures spéciales d'accès à la citoyenneté française pour chaque territoire (1909 pour Madagascar, 1881 pour la Cochinchine, 1912 pour l'A.-O. F. et l'A.-É. F., 1932 pour la Nouvelle-Calédonie). Pour les étrangers ayant émigré dans l'Empire français, le décret du 7 février 1897 étend l'application de la législation sur la nationalité de 1889 .

31 Dans cette situation complexe, comme la « loi » de 1940 a-t-elle été appliquée ? 
32 À partir de la base Dénat, nous avons donc recensé 188 personnes nées dans les colonies françaises, protectorats ou territoires sous mandat, mais sans spécification sur leur statut : indigène, protégé ou étrangers immigrés.

De plus, si le texte de la «loi» du 22 juillet 1940 ne mentionne pas le territoire d'application, il entend réviser toutes les acquisitions de nationalité française, ce qui semble exclure le cas des indigènes des colonies, puisqu'ils étaient déjà français ! La base Dénat ne permet pas de répondre de façon systématique à cette question. En isolant les individus nés dans l'Empire, quelques très rares cas semblent concerner des colonisés, touchés par la procédure de retrait.

35 Ainsi Fatima Bent El Houssine Ben Ali, épouse Dantes, née à Marrakech et habitant à Oujda (Maroc), qui fait l'objet d'un retrait de nationalité lors du premier décret de retrait le $1^{\text {er }}$ novembre 1940. De nationalité marocaine, elle appartient au groupe des protégés français. Son dossier de naturalisation porte la mention « devenue française par son mariage le 28 juin 1939 ».

Un autre cas attire notre attention: Duong Van Giao, né en 1888 à Daphuoc (Cochinchine), perd sa nationalité en 1944.

37 Afin de comprendre précisément quel est le sort des populations colonisées qui ont obtenu la naturalisation, nous avons décidé de reconstituer le travail de la commission en pratique, en étudiant de façon fine et intensive les dossiers de naturalisation euxmêmes. Pour cela, il s'agit de comprendre comment étaient traités les dossiers des colonisés naturalisés en les comparant aux autres dossiers ouverts pendant les mêmes séances d'instruction. Ce travail, que nous sommes actuellement en train de mener, est encore inachevé.

\section{Un nouveau mystère : la « loi » du 17 avril 1942}

38 Parallèlement à cette première recherche à partir de la base Dénat et poursuivie avec l'étude systématique des dossiers de naturalisation, nous travaillons sur un texte quasiment jamais mentionné : celle de la « loi » du 17 avril 1942 .

39 Ce texte, publié au Journal officiel ${ }^{10}$, est totalement absent des principales études sur la nationalité française ou les dénaturalisations de Vichy ou sur la nationalité dans les colonies. Il est juste mentionné dans le livre d'Eric Jennings Vichy sous les tropiques ${ }^{11}$. Nous avons donc décidé de la reproduire ici dans son intégralité.

Loi du 17 avril 1942 :

Art. 1. Il sera procédé à la révision de toutes les admissions aux droits de citoyens français des anciens indigènes, sujets protégés ou administrés sous mandat français originaires d'un territoire relevant du secrétariat d'État aux colonies, qui, depuis le $1^{\mathrm{er}}$ septembre 1939, ont été l'objet soit d'une mesure d'internement administratif, soit d'une condamnation par une juridiction de droit commun ou un tribunal d'exception pour menées antifrançaises ou crime ou délit de droit commun.

Art. 2. Le retrait des droits de citoyen sera, s'il y a lieu, prononcé par décret pris sur le rapport du garde des sceaux, ministre secrétaire d'État à la Justice, et du secrétaire d'État aux Colonies, après avis de la commission prévue par l'article 2 de la loi du 22 juillet 1940 relative à la révision des naturalisations.

Ce décret fixera la date à laquelle remontera la perte de la qualité de citoyen français.

Cette mesure pourra être étendue à la femme et aux enfants de l'intéressé. 
Art. 3. L'individu privé de la qualité de citoyen français en vertu de la présente loi est replacé dans le statut indigène qu'il possédait ou que possédait son auteur avant admission aux droits de citoyen.

Art. 4. Le présent décret sera publié au Journal officiel et exécuté comme loi de l'État.

La « loi » de 1942 est rédigée selon le même modèle que celle de 1940 qui est d'ailleurs mentionnée explicitement dans son article 2.

Elle annonce la révision des admissions au droit de citoyens de tous les sujets coloniaux, quel que soit leur statut: indigène, protégé ou administrés sous mandats, avec la seule restriction d'être l'objet d'une mesure administrative ou judiciaire.

Comme dans la « loi » de 1940, la mesure peut être étendue à sa famille de l'individu objet du retrait.

En revanche, il n'est pas fait de mention de la loi du 10 août 1927, ni d'une quelconque limitation temporelle ou chronologique dans son champ d'application, et de fait, elle concerne des naturalisations du début des années 1920.

4 Cependant, la signification et la portée de ce texte demeurent ambiguës. D'un côté, il annonce une vaste action de révision similaire à celle de 1940, ce qui semble suggérer la volonté d'inclure les sujets coloniaux dans le mouvement de révision alors qu'ils en avaient été écartés pendant un an. D'ailleurs, c'est sans doute la promulgation de ce texte qui explique l'inclusion, au sein de la Commission de révision, d'un représentant du secrétariat des Colonies.

5 Cependant jusqu'à présent, notre analyse de la base Dénat ou des cartons de naturalisation n'ont pas confirmé qu'une telle entreprise de révision systématique ait été menée à bien.

Au contraire, les seuls retraits de citoyenneté que nous avons trouvés à la suite de la « loi » du 17 avril 1942 sont ceux mentionnés par Eric Jennings: Jules Ranaivo (Madagascar), Duong Van Giao et Phan Van Thiet (Indochine), trois militants engagés dans la cause indépendantiste.

C'est pourquoi Eric Jennings a suggéré que la « loi » de 1942 était un mélange du texte du 22 juillet 1940 avec celui du 23 juillet 1940 sur la déchéance de nationalité à l'encontre de ceux qui avaient quitté le territoire français. La restriction de l'application de la «loi » aux sujets coloniaux faisant déjà l'objet d'une mesure répressive de l'administration coloniale semble confirmer la signification avant tout politique (dans le sens de répressif du terme) du projet.

48 L'étude des dossiers confirme cette impression. Dans les deux cas de Ranaivo et Van Giao, la procédure commence avec la transmission d'un rapport secret du secrétariat aux Colonies au ministre de la Justice (direction des affaires politiques, $5^{\mathrm{e}}$ bureau) informant de la demande de l'administration coloniale (haut-commissaire pour Van Giao, gouvernement général pour Ranaivo) visant à les dénaturaliser.

49 À chaque fois, les autorités coloniales joignent à leur demande un rapport détaillé sur le danger politique supposément représenté par ces individus.

La commission, qui a étudié ces deux demandes le même jour, semble avoir simplement recopié les arguments de l'administration coloniale pour justifier sa décision de retrait. Il convient, néanmoins, de continuer l'enquête afin de déterminer s'il n'existe pas d'autre cas, qui pourraient ne pas figurer d'ailleurs dans la base Dénat, si celle-ci était limitée à l'application de la « loi » du 22 juillet 1940. 
51 En conclusion, cette communication n'est qu'une première étape d'une recherche encore à approfondir, à la fois pour reconstituer le travail de la commission de révision des naturalisations sur les dossiers de naturalisation de colonisés (et plus largement des naturalisations prononcées dans l'Empire). Par ailleurs, à partir des archives des administrations coloniales (en particulier des archives du gouvernement général à Hanoï), il nous faut encore déconstruire la genèse de la « loi » du 17 avril 1942 pour mieux en comprendre la portée et son application pratique.

\section{NOTES}

1. ZALC Claire, Dénaturalisés. Les retraits de nationalité sous Vichy, Paris, Seuil, 2016.

2. BLÉVIS Laure, «La citoyenneté française au miroir de la colonisation : étude des demandes de naturalisation des "sujets français" en Algérie coloniale ", Genèses, vol. 53, n 4, 2003, p. 25-47.

3. À l'exception de JENNINGS Eric, Vichy sous les tropiques. La Révolution nationale à Madagascar, en Guadeloupe, en Indochine, 1940-1944, Paris, Grasset, 2001.

4. Il ne s'agit pas d'une loi votée par le Parlement puisque l'Assemblée est dissoute par Pétain le 10 juillet 1940 ; c'est pourquoi nous préférons indiquer ce terme, qui figure dans le texte entre guillemets.

5. ZALC Claire, ibid, p. 70.

6. LE FOLL-LUCIANI Pierre-Jean, Les juifs algériens dans la lutte anticoloniale. Trajectoires dissidentes (1934-1965), Rennes, Presses universitaires de Rennes, 2015.

7. BLÉVIS Laure, "En marge du décret Crémieux. Les Juifs naturalisés français en Algérie (1865-1919) », Archives juives, 2012/2, vol. 45, p. 47-67.

8. ZALC Claire, ibid, ; POINSOT Annie, "Retrait, maintien, enquête». La Commission de révision des naturalisations (1940-1944). Un instrument de la politique xénophobe et antisémite de Vichy?, mémoire de master 2, université Paris 1-Panthéon-Sorbonne, octobre 2013.

9. Merci à Aliénor Cadiot de nous avoir signalé son existence. Cette loi est également mentionnée dans un rapport secret de février 1944 du Comité français de libération nationale concernant un projet de loi de déchéance (Archives nationales, BB/30/1731).

10. Journal officiel de la République française, 13 juin 1942, p. 2058.

11. JENNINGS Eric, Vichy sous les tropiques. La Révolution nationale à Madagascar, en Guadeloupe, en Indochine, 1940-1944, Paris, Grasset, 2001. 


\section{AUTEURS}

LAURE BLÉVIS

Université Paris Nanterre-ISP

\section{CLAIRE ZALC}

Institut d'histoire moderne et contemporaine 


\title{
Les dossiers de dénaturalisation, nouvelle source pour l'histoire de la déportation en France
}

\author{
Karen Taieb
}

Ce média ne peut être affiché ici. Veuillez vous reporter à l'édition en ligne http:// books.openedition.org/pan/1230

\section{La base des victimes de la Shoah en France}

2 À l'origine, la base de données des victimes de la Shoah en France a été réalisée pour permettre l'élaboration du Mur des noms, monument commémoratif qui marque l'entrée du Mémorial et sur lequel sont gravés les noms des près de 76000 personnes déportées de France en tant que juives entre 1942 et 1944.

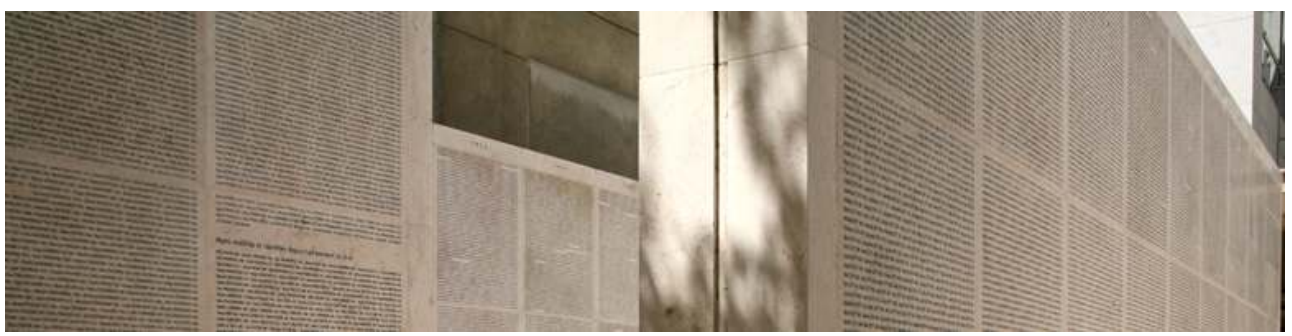

Le Mur des noms (crédit : Mémorial de la Shoah).

3 Nous avons entamé nos travaux sur cette base de données en 1999 y travaillons encore aujourd'hui , je dirais même - plus que jamais.

4 En l'occurrence, quand les Archives nationales ont sollicité le Mémorial de la Shoah pour savoir dans quelle mesure il serait possible d'accéder à sa base de données pour déterminer plus facilement et plus rapidement lesquels des dénaturalisés avaient été 
déportés, nous sommes rapidement convenus que nous devions échanger nos données. J'aimerais insister sur ce point et la question du partage des informations.

5 Nous travaillons, nous gérons des corpus de données sensibles certes, mais qui, admettons-le, ne relèvent pas la sûreté nationale, mais de la vie privée des personnes. Il nous est souvent demandé pourquoi nous ne mettons pas tout en ligne : les bases de données, les documents numérisés, etc. Sans entrer dans des considérations juridiques ou même éthiques, je dirai seulement qu'il nous est possible de partager des informations, croiser des bases de données, dans le respect de la vie privée des personnes et dans le cadre d'un projet pertinent, pour les rendre accessibles par des voies d'accès multiples, comme dans le cas qui nous intéresse aujourd'hui, aux Archives nationales et au Mémorial de la Shoah.

6 Nous sommes ainsi parvenus, grâce au croisement de nos bases de données respectives, à identifier près de 750 personnes qui ont fait l'objet d'une mesure de dénaturalisation et qui ont également été déportées.

7 Le travail réalisé par Bernard Raquin permet notamment au chercheur, quand il consulte la base Dénat aux Archives nationales et qu'il trouve une personne qui a été déportée, de pointer sur la page du site internet du Mémorial et d'accéder aux informations dont nous disposons sur cette personne, complémentaires de ce que l'on peut trouver dans son dossier de naturalisation conservé aux Archives nationales. Le chercheur aura ainsi accès aux informations sur la personne, à la liste du convoi sur laquelle figure son nom, à la fiche de fouille à Drancy si elle existe, peut-être à une photographie et à des documents associés.

8 Pour résumer, à partir du point d'entrée de la base Dénat pour une personne, le lien permet l'ouverture et l'accès à d'autres informations sur cette personne.

9 À l'inverse, une personne qui viendra au Mémorial faire une recherche sur une personne déportée se verra indiquer si cette personne a fait l'objet d'une mesure de retrait de la nationalité et ainsi savoir, si elle le souhaite, qu'elle pourra consulter le dossier sur place aux Archives nationales, voire bientôt directement en version dématérialisée au Mémorial de la Shoah ou sur les sites des Archives nationales.

\section{Les informations contenues dans les dossiers}

10 Quel intérêt présentent ces dossiers pour les chercheurs qui travaillent sur les Juifs déportés de France ou qui s'y intéressent?

11 Les dossiers de demande de naturalisation sont de ces archives purement administratives au moment de leur constitution qui nous apportent aujourd'hui une multitude de détails sur les personnes concernées. Ces dossiers et les documents qu'ils contiennent sont un lien direct avec la personne qui postule à la naturalisation. Elle doit justifier son attachement à la France, motiver sa demande et révéler bien des détails sur sa situation familiale ou ses conditions de vie. Bien entendu, comme toujours, les documents administratifs sont une source parmi d'autres, qu'il convient d'ailleurs de contredire, de critiquer, de confronter. Évidemment, le postulant à la naturalisation doit convaincre, rassurer. Il est dans la position délicate du demandeur. Les renseignements qu'il donne, hormis ceux qui sont strictement biographiques, sont à prendre avec précaution. Il n'en est pas moins vrai qu'au travers de ces archives, ce sont la voix et la main de celui qui remplit le dossier qui nous apparaissent. 

initiale de la demande de naturalisation qui nous apprend des informations importantes; le retrait de nationalité, qu'il aboutisse ou non, renseigne peu sur la personne, sauf si elle fait un recours. Ces dossiers-là nous renseignent davantage sur les travaux de la commission de révision, sur la procédure de dénaturalisation et les motifs invoqués pour le retrait. Le travail de Claire Zalc ${ }^{1}$ est exemplaire et dit tout sur ces aspects. négligeable de dossiers de Juifs qui ont été déportés. L'idéal serait de pouvoir identifier les dossiers de tous les Juifs déportés naturalisés et qui n'ont pas l'objet d'une dénaturalisation. Cela permettrait alors de connaître plus précisément la population des Juifs naturalisés qui non pas été déportés et d'en comprendre peut-être les raisons.

Prenons l'exemple d'Abraham Pikovsky. Il est arrivé en France en 1905, venant de Russie où il était né en 1896. Il exerce la profession de chauffeur d'automobiles. Il acquiert la nationalité française en 1937. Il est marié à Brunette, elle-même française, et ils ont eu ensemble quatre enfants, tous français nés en France: Annette, née le 3 février 1926, Louise, le 7 décembre 1927, Jean, le 10 septembre 1929 et Lucie, le 16 mai 1932. La famille vit à Boulogne-Billancourt, en banlieue parisienne.

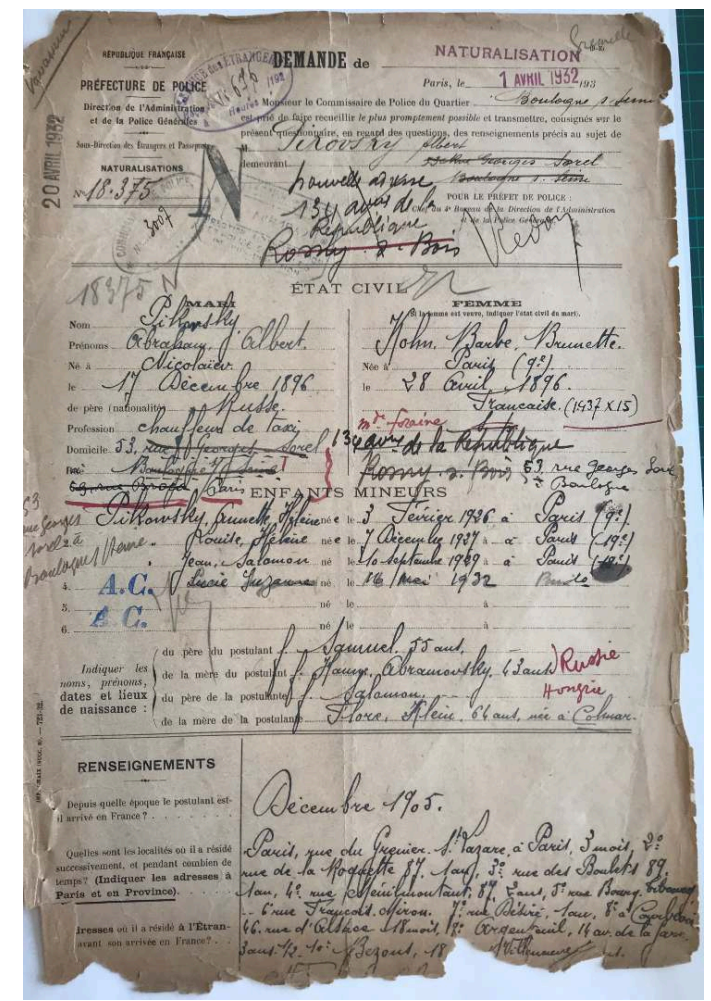

Première page de la feuille d'instruction du dossier de demande de naturalisation d'Abraham Pikovsky (Archives nationales, 19770291/8, dossier $39237 \times 36$ ).

Dans le dossier de naturalisation d'Abraham, on trouve des attestations de ses employeurs et des maires de ses communes de résidences successives, qui permettent de retracer son parcours de manière assez précise. Mais elles n'empêchent pas qu'il fasse l'objet d'une procédure de retrait de dénaturalisation. Le décret de retrait est daté du 21 mars 1941. Cette procédure vise seulement Abraham, pas le reste de la famille. Pourtant, en janvier 1944, c'est la famille entière qui est prise au piège. L'histoire de 
Louise Pikovsky, l'un des filles d'Abraham, élève au lycée La Fontaine, est connue depuis peu. Louise est une lycéenne brillante, une jeune fille pleine de vie, qui entretient une correspondance avec sa professeure de lettres, $\mathrm{M}^{\text {elle }}$ Malingrey. C'est à elle qu'elle adresse un ultime message, le 22 janvier 1944, le jour de son arrestation. Ce message ainsi que d'autres lettres et photographies ont été retrouvés à l'occasion d'un déménagement au sein du lycée. Les documents étaient restés au fonds d'une armoire ; ils ont été versés aux Mémorial de la Shoah².

16 Est-ce que la dénaturalisation d'Abraham a eu un impact sur son arrestation? La nationalité française de sa femme et de ses enfants ne les a malheureusement pas protégés.

\section{Les recours}

Parmi les Juifs déportés qui se sont vu retirer leur nationalité française, un certain nombre a déposé un recours. La plupart de ces recours n'ont pas abouti. Mails il est intéressant de se plonger dans ces dossiers pour voir de quelle manière les choses étaient présentées par les postulants. Je prendrai deux exemples.

En premier lieu, celui de Moszek Papentreger qui obtient sa naturalisation en 1937 après avoir entamé les démarches en 1932. Il se voit retirer sa nationalité française en 1941, mais, ne pouvant se résoudre à cette situation, il dépose un recours gracieux. Il écrit lors une lettre au garde des Sceaux, le 8 janvier 1942. Cette lettre se trouve dans le dossier. Il y exprime son incompréhension devant la mesure qui le frappe: "J'avais quatre enfants quand j'ai été naturalisé, j'en ai huit aujourd'hui [...] j'étais marchand ambulant mais, en raison des nouvelles lois, je ne peux plus faire les marchés alors je me suis engagé comme bûcheron. Je ne gagne pas beaucoup mais cela me permet de nourrir mes enfants ». À aucun moment Moszek ne dit qu'il est juif, que les nouvelles lois dont il parle sont celles qui mettent les Juifs au ban de la société. En 1941, le retrait de la nationalité a d'abord visé Moszek, son épouse et leurs quatre enfants les plus grands. Mais l'administration n'en reste pas là, et les quatre plus jeunes sont à leur tour dénaturalisés, en septembre 1943. Moszek ne le saura pas, car il avait été déporté, le 22 mars 1943, par le convoi 52 à destination du camp d'extermination de Sobibor.

Prenons un second exemple : le cas d'Aron Rozenbaum. Né le $1^{\text {er }}$ février 1911, il a été naturalisé français le 15 décembre 1939. Il travaille la fourrure à Tours (Indre-et-Loire). Il semble bien intégré dans la moyenne bourgeoisie de la ville et jouit d'une excellente réputation. Marié, père d'une enfant et en attente d'un second, il se voit lui aussi retirer sa nationalité française, le 14 juin 1941. Il écrit une lettre de recours qui est conservée dans son dossier. On y trouve également une pétition signée par près de vingtcinq personnes qui toutes attestent de sa probité. Aron obtient gain de cause, sa nationalité lui est rendue en janvier 1942 : il restera français. Cela ne le protègera pas. Il est arrêté et déporté par le convoi 73, le 13 mai 1944.

\section{Conclusion et perspectives}

En conclusion, je dirai que ce corpus n'est certainement pas fondamental pour l'étude sur la déportation des Juifs de France, mais il est une nouvelle source d'information pour les 750 déportés identifiés et, nous le savons, les personnes qui font des 
recherches sur le parcours de vie sont avides de documentation supplémentaire, dont la moindre bribe est une trouvaille.

21 La deuxième chose qui me tient à cœur, c'est de souligner la facilité avec laquelle nos institutions coopèrent. Nous sommes actuellement en phase de préparation de la mise en œuvre du partenariat auquel je joint le musée de l'Holocauste des États-Unis et qui vise à la numérisation des dossiers qui sont passés devant la commission de révision. Le projet devrait être réalisé en 2019. Les dossiers seront ainsi consultables dans nos salles de lecture respectives. ${ }^{3}$

\section{NOTES}

1. ZALC Claire, Dénaturalisés. Les retraits de nationalité sous Vichy, Paris, Seuil, 2016.

2. TROUILLARD Stéphanie, Si je reviens un jour, 2018 [web-documentaire hébergé par France 24].

3. Voir plus bas l'épilogue d'Élisa Dupuis.

\section{AUTEUR}

\section{KAREN TAIEB}

Responsable du service des archives du Mémorial de la Shoah 


\section{Épilogue}

\section{Élisa Dupuis}

1 La journée d'étude consacrée à la base Dénat a permis la présentation des travaux menés par les acteurs qui se sont investis dans sa création et dans son utilisation. Elle fut une nouvelle occasion de voir se manifester la complémentarité des dimensions documentaire, analytique et mémorielle du travail que mènent archivistes et chercheurs. Elle fut également la démonstration que mutualiser les savoir-faire et les expertises de chacun permet toujours de porter un regard plus nuancé sur l'histoire, à différentes échelles, notamment géographiques et sociologiques. On ne peut donc qu'aspirer à la perpétuation et la diversification de ces dialogues entre les disciplines, entre les investigations et entre ceux qui en prennent la charge. C'est un enjeu de transmission auquel tend à contribuer l'opération récolement avant numérisation des dossiers de dénaturalisés présentée ci-après.

2 À l'initiative des Archives nationales et en partenariat avec le Mémorial de la Shoah de Paris et le United States Holocaust Memorial Museum, a débuté en février 2019 une opération de grande ampleur visant à numériser les 8130 dossiers des 15195 « dénaturalisés de Vichy ». À l'horizon 2022, les dossiers seront mis à disposition $\mathrm{du}$ public, en salle de lecture, via les plateformes informatiques internes des organismes pilotes. Trois envois successifs, d'une centaine de milliers de vues chacun, seront effectués par les Archives nationales à un prestataire de numérisation. Le chantier de récolement de ces dossiers est réalisé sur le site de Pierrefitte-sur-Seine auprès du département de la Justice et de l'Intérieur.

3 Il s'est agi tout d'abord de confronter les numéros de dossiers répertoriés dans la base Dénat aux tables de correspondances qui inventorient les cotes associées. La première vague concerne la sous-série $\mathrm{BB} / 11$ qui représente 2500 dossiers de naturalisation ouverts entre 1910 et 1930, comportant les mesures de retrait prononcées à partir de juillet 1940. Les versements des dossiers postérieurs à 1930, identifiés en cotation continue, seront traités durant les vagues suivantes. Le fichier de récolement ainsi édité permet la localisation en magasin et l'extraction de chaque dossier.

4 Les dossiers extraits reçoivent ensuite deux traitements successifs: un reconditionnement matériel (prélèvement des éléments métalliques, dépliage des feuillets, dépoussiérage, chemisage), indispensable étant donné la fragilité de la plupart 
des dossiers ; et un foliotage, procédé chronophage mais qui présente l'avantage, entre autres, au vu de la multitude de documents, d'un suivi systématique pièce à pièce. Il permet de baliser non seulement le protocole de numérisation à venir, mais aussi le rattachement ultérieur des images aux vues du dossier correspondant.

Ce traitement matériel est aussi une phase qui révèle la singularité des dossiers et, partant, celle de l'histoire personnelle des individus. Ils sont généralement structurés en deux ensembles distincts: la demande de naturalisation et la procédure de déchéance (pour les demandes instruites avant 1927) ou de retrait (pour les demandes instruites depuis 1927). De nombre inégal, les documents constitutifs sont de nature diverse : pièces d'état civil, certificats de résidence, de scolarité, extraits de casier judiciaire et procès, attestations d'emploi, de services (militaires) rendus, motifs officiels du retrait, mais aussi lettres, parfois manuscrites, de recommandation, de motivation, de recours, de correspondance familiale ou administrative, photographies.

6 Pour des raisons réglementaires, un examen minutieux des pièces a été mené afin de repérer des documents dont le délai de communicabilité n'est pas encore échu' ${ }^{1}$. Cette nécessité a été l'opportunité d'appréhender systématiquement la richesse de ce corpus captivant.

7 Cette opération menée à son terme rendra accessible au plus grand nombre l'histoire de ces citoyens français que sont "les dénaturalisés de Vichy». Cette perspective patrimoniale, qui portera à la connaissance les particularités de chaque cas, espère aussi donner un accès et une voix aux descendants biologiques ou spirituels de ceux qui ont été confrontés aux injonctions juridiques du régime de Vichy et à leurs répercussions effectives et morales.

Comme le rappelle Laurent Joly à l'occasion de la présentation de son livre L'État contre les juifs. Vichy, les nazis et la persécution antisémite: «Ce que l'on peut apprendre de l'enquête documentaire sur l'Histoire est que la constitution de celle-ci n'est faite que de problématiques jouées à l'échelle de l'individu, dont l'étude doit être faite assidûment. $\aleph^{2}$. Il apparaît en ce sens que la base Dénat constitue un potentiel certain pour les nombreux aspects de la recherche qu'il reste à développer.

\section{NOTES}

1. Pour la communicabilité des archives publiques, $c f$. les articles L213-1 à 8 du code du patrimoine; pour la réglementation récente de la diffusion des données, $c f$. RICARD Bruno, « La diffusion des archives sur Internet : de nouvelles règles ", Droit(s) des archives, 15 février 2019.

2. Paroles d'histoire, 2018, podcast animé par André Loez, émission du 31 octobre 2018 [https:// parolesdhistoire.fr/index.php/2018/10/31/27-persecution-et-survie-des-juifs-sous-vichy-aveclaurent-joly-et-jacques-semelin-31-oct/]. 


\section{AUTEUR}

\section{ÉLISA DUPUIS}

Chargée du récolement avant numérisation des dossiers de dénaturalisés aux Archives nationales (Mémorial de la Shoah) 